\title{
Mobile Node Localization via Pareto Optimization: Algorithm and Fundamental Performance Limitations
}

\author{
Alessio De Angelis, Member, IEEE, Carlo Fischione, Member, IEEE
}

\begin{abstract}
Accurate estimation of the position of network nodes is essential, e.g., in localization, geographic routing, and vehicular networks. Unfortunately, typical positioning techniques based on ranging or on velocity and angular measurements are inherently limited. To overcome the limitations of specific positioning techniques, the fusion of multiple and heterogeneous sensor information is an appealing strategy. In this paper, we investigate the fundamental performance of linear fusion of multiple measurements of the position of mobile nodes, and propose a new distributed recursive position estimator. The CramérRao lower bounds for the parametric and a-posteriori cases are investigated. The proposed estimator combines information coming from ranging, speed, and angular measurements, which is jointly fused by a Pareto optimization problem where the mean and the variance of the localization error are simultaneously minimized. A distinguished feature of the method is that it assumes a very simple dynamical model of the mobility and therefore it is applicable to a large number of scenarios providing good performance. The main challenge is the characterization of the statistical information needed to model the Fisher information matrix and the Pareto optimization problem. The proposed analysis is validated by Monte Carlo simulations, and the performance is compared to several Kalman-based filters, commonly employed for localization and sensor fusion. Simulation results show that the proposed estimator outperforms the traditional approaches that are based on the extended Kalman filter when no assumption on the model of motion is used. In such a scenario, better performance is achieved by the proposed method, but at the price of an increased computational complexity.
\end{abstract}

Index Terms-Sensor Fusion, Positioning, Distributed Models, Networks, Optimization, Cramér Rao Lower Bound.

\section{INTRODUCTION}

In many sensor network applications it is desirable to accurately estimate the position of mobile wireless nodes [1]. Relevant applications are vehicular traffic monitoring, asset tracking, process monitoring, and control of autonomous agents. As an example, accurate position information is crucial for emergency personnel and first responders, see, e.g., [2]. The commonly used Global Navigation Satellite Systems (GNSSs) provide position information with an accuracy of about 3 to $10 \mathrm{~m}$ in outdoor scenarios. However, in indoor and electromagnetically-challenged environments, such as urban canyons or forests, the coverage provided by GNSSs is not sufficient. Therefore, for such environments, several alternative

Alessio De Angelis is with the Department of Engineering, University of Perugia, Italy. E-mail: alessio.deangelis@unipg.it. Carlo Fischione is with ACCESS Linnaeus Centre, Department of Electrical Engineering, KTH Royal Institute of Technology, Stockholm, Sweden. E-mail: carlofi@kth.se positioning technologies have been developed, particularly for wireless indoor localization; for an extensive review see [3] and references therein.

The topic of localization using wireless signals has received much attention in many different research areas. Numerous solutions and applications have been proposed, including distributed algorithms for wireless sensor networks [4], the robust distributed method proposed in [5], and the technique for passive device-free sensor localization proposed in [6]. The key aspect of cooperation between the nodes of a wireless network for localization purposes has been investigated in [7], and experimentally evaluated in [8]. The fundamental importance of position information for nodes in wireless sensor networks is highlighted in [9], where topology-aware estimation algorithms are developed, using techniques from the spectral graph theory field. In this context, distributed gossip algorithms for sensor networks localization have been studied in [10] and [11]. In particular, [10] shows that kernel averaging techniques for localizing an isotropic source based on measurements by distributed sensors may provide improved robustness and accuracy than least squares estimators. Moreover, in [11], a distributed algorithm is proposed and characterized, which estimates the positions of nodes given a minimal number of anchors and using only data-exchange between neighboring nodes.

The robustness with respect to range-measurement outliers due to non-line of sight conditions has been analyzed in [12], which introduces a robust extended Kalman filter for locating a mobile terminal node in wireless networks. Moreover, in [13], a Bayesian algorithm is proposed for self-localization and tracking of a mobile node through ranging with knownposition anchors. The algorithm is robust to NLOS propagation by combining a Markov model for sight-state estimation and a particle filter for location estimation, with a simple general motion model. Experiments using the IEEE 802.15.4a chirp-spread-spectrum ranging technology show accuracy of approximately $2 \mathrm{~m}$ in an indoor environment with varying NLOS and LOS conditions. In [14], the concept of schedulebased network localization is introduced, which enables selflocalization of mobile nodes, as well as localization of the entire network, without communication overhead.

In the context of wireless short range indoor positioning, Pulse-based Ultra-Wideband (UWB) techniques are of particular interest due to numerous good qualities, such as a fine time resolution (which allows for a ranging accuracy of the order of centimeters when used in conjunction with time-of-arrival 
methods) a resilience to multipath propagation effects of the wireless channel, and a low-power device implementation [15]. Recent results on the fundamental limits for wideband radio localization of static nodes have been presented in [16]. For the case of mobile cellular systems, a performance analysis has been presented in [17]. The fundamental limitations of mobile localization have been extensively studied in [18] for bearings-only measurements in the target tracking field, and in [19] in the terrain-aided navigation field. In the context of sensor networks node localization, the Cramér-Rao lower bound (CRLB) has been evaluated for the case of receivedsignal-strength (RSS) range measurements in [20] and in [21]. Therein, the RSS model has been linearized with only ranging measurements and without using any velocity and orientation information.

To improve the performance of a positioning system in terms of reliability of the estimates (integrity), accuracy, and availability, it is appealing to process information obtained from a number of sensors by means of fusion techniques [22], [23]. These techniques involve the processing of different information sources to overcome the fundamental limitations of sensor measurements such as GNSSs, inertial navigation systems (INSs), odometry, and local radio technologies. An extensive survey of the most common information sources and sensor fusion approaches is provided in [24] in the context of automotive positioning. Concerning information fusion for UWB, the commonly employed techniques are based on the extended Kalman filter (EKF), [25], or other non-linear filtering approaches such as particle filters methods [26]. In [27], a 6 degrees-of-freedom tracking system is presented, which performs sensor fusion on a UWB distance-measuring device and an inertial sensor consisting of triaxial accelerometers and gyroscopes. In [28], an UWB sensor fusion technique based on the complementary filter approach is presented, where the error states are estimated by an EKF using the UWB ranging measurements. Subsequently, the estimated errors are fed back to correct for the inertial navigation system biases, which would otherwise grow unbounded.

In this paper, we investigate a novel sensor fusion technique, based on Pareto optimization, for node self-localization using heterogeneous information sources. It is assumed that a node wishing to estimate its own position has available ranging, speed and orientation information. We stress here that the problem that we consider in this paper is self-localization, which is fundamentally different from the target tracking problem that is widely studied in the signal processing literature, see e.g. [29]. Furthermore, the fundamental performance limitation of the linear fusion is investigated in this paper. This investigation is a substantial extension of our earlier work in [30] and [31], where the performance of the Pareto optimization were not fully investigated and no derivation of the CRLB were considered. The posterior CRLB [32] and the parametric CRLB [19] are characterized for both any generic trajectory of the mobile node and for a specific trajectory. Since ranging information gives an error with low bias and a high variance, and measurements of the speed and absolute orientation (dead-reckoning) give an error with a low variance and a high bias, the overall information is jointly processed to overcome the individual limitations of ranging, speed and orientation measurements. By numerical simulations and by evaluating the CRLB, we show that our new method may outperform existing solutions, including several commonly employed techniques based on the Kalman filter. However, the price payed is a higher computational complexity.

This paper has several distinguished features compared to the related work mentioned above. Specifically, our system model is original because the mobile node position estimation is performed by ranging measurements, with respect to fixed position nodes, together with velocity and orientation measurements without any linearization or simplification. For the derivation of the estimator, we assume a very simple dynamical model of the movement of the mobile node. This model allows for flexibility, robustness, low-complexity implementation and works well in several different scenarios, as we show later. Only local processing at such a node is used, thus enabling a completely distributed strategy. Furthermore, the estimation is performed in a cooperative fashion, in the sense that operation of the estimator at the mobile node relies on the cooperation of fixed-position nodes acting as responders during ranging measurements.

Therefore, our method is of a general nature and can be applied to any motion scenario. Compared to, e.g., [20] and [21], we investigate the CRLB without any linearization of the ranging model and we consider simultaneously ranging, velocity and orientation measurements, which were not considered therein. Moreover, in this paper, we address one of the main challenges for developing sensor fusion methods, namely the statistical characterization of the estimation error. Based on this characterization, a sensor fusion method is derived by solving a Pareto optimization problem, where a tradeoff between the variance of the estimation error and its bias is exploited. The Pareto optimization approach was first proposed in our initial study in this context in [31].

The remainder of this paper is organized as follows: The problem is formulated in Section II. Then, the proposed sensor fusion method is derived in Section III. Furthermore, the fundamental limits of an estimator that fuses ranging, velocity and orientation measurements are investigated in Section IV by means of the CRLB. Numerical simulation results are presented in Section V. Finally, conclusions are drawn in Section VI.

\section{A. Notation}

We denote real $n$-dimensional vectors with lowercase boldface letters, such as $\mathbf{x}$, and $\mathbb{R}^{n \times m}$ matrices with uppercase boldface letters, such as $\mathbf{A}$. The superscript $(\cdot)^{T}$ indicates the transpose of a matrix. Given two matrices $\mathbf{A}$ and $\mathbf{B}$, the inequality $\mathbf{A} \preceq \mathbf{B}$ denotes that the matrix $\mathbf{B}-\mathbf{A}$ is positive semidefinite. Given a scalar function $f(\mathbf{x}): \mathbb{R}^{n} \rightarrow \mathbb{R}$, $\nabla_{\mathbf{x}} f(\mathbf{x})=\left[\frac{d f(\mathbf{x})}{d x_{1}}, \ldots, \frac{d f(\mathbf{x})}{d x_{n}}\right]^{T}$. Given a vector function $\mathbf{f}(\mathbf{x}): \mathbb{R}^{n} \rightarrow \mathbb{R}^{n}$, we use the gradient matrix definition $\nabla_{\mathbf{x}} \mathbf{f}(\mathbf{x})=\left[\begin{array}{lll}\nabla f_{1}(\mathbf{x}) & \ldots & \nabla f_{n}(\mathbf{x})\end{array}\right]$, which is the transpose 


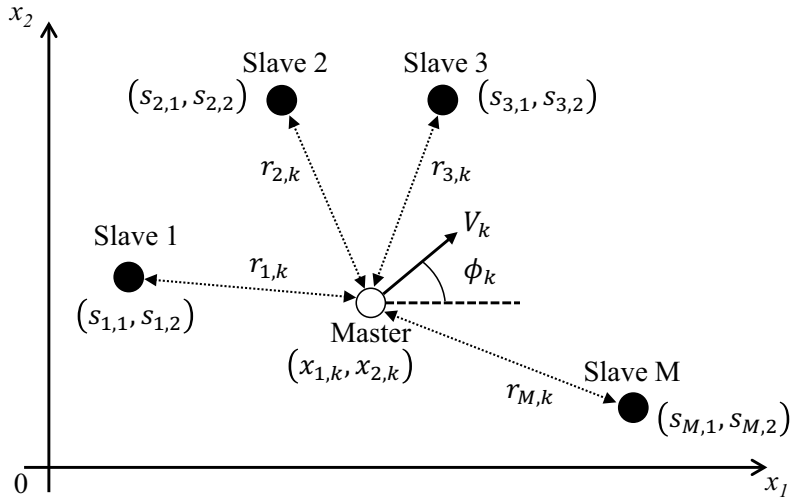

Fig. 1. Model of the localization system. The slave nodes are placed at fixed and known positions. At time $k$, the unknown-position master node measures its distance $r_{i, k}$ with respect to each slave $i$ by measuring the round-trip-time of UWB pulses. Speed $V_{k}$ and orientation $\phi_{k}$ of the master are measured using on-board sensors.

of the Jacobian matrix. We indicate with $\tilde{\mathbf{x}}$ a noisy measurement of $\mathbf{x}$ and with $\hat{\mathbf{x}}$ an estimator of $\mathbf{x}$. The statistical expectation operator is denoted by $\mathbb{E}\{\cdot\} \cdot p(\mathbf{x})$ denotes a probability density function (pdf) of the random vector $\mathrm{x}$.

\section{Problem FORMULATION}

Consider a system where a mobile node, which we denote as master, needs to estimate its own planar position $\mathbf{x}_{k} \in \mathbb{R}^{2}$, with $\mathbf{x}_{k}=\left[\begin{array}{ll}x_{1, k} & x_{2, k}\end{array}\right]^{T}$, where $k$ is the discrete time index. To do so, the master measures its distance with respect to $M$ devices, which we denote as slaves, with $M \geq 4$. We do not assume any a-priori information on the mobility of the master. It is allowed to move by following a linear trajectory with constant velocity as well as a random trajectory with random acceleration. An example of such a system is provided by the in-house developed experimental platform that has been characterized in [28], [33], where the distance measurement is obtained by means of the round-trip-time of UWB signals. This distance measurement approach, also known in the literature as two-way time-of-arrival, avoids the need for synchronization between mobile node and slaves, therefore allowing for asynchronous operation and reduced infrastructure requirements. Alternative approaches, such as one-way time-of-arrival and time-difference-of-arrival, suffer from clock skew and require additional infrastructure for precise clock synchronization [34].

We assume that the master is equipped with UWB transmission and sensors that measure its speed and absolute orientation. As an example, speed sensors might be implemented by means of odometry in vehicular or mobile-robotics applications, such as those modeled in [35]. Furthermore, information about absolute orientation could be obtained from a heading sensor, for example a compass or magnetometer, as in [36]. The diagram in Fig. 1 shows a representation of the system model. Furthermore, we assume that the slaves are located in fixed and known positions that are denoted as $s_{i, j}$, with $i=1, \ldots, M$ and $j=1,2$. We denote the ranges, namely the distances between the master and each slave at time instant $k$, by $r_{i, k}, i=1, \ldots, M$. The measurements of the ranges, denoted as $\tilde{r}_{i, k}$, are affected by additive zero-mean Gaussian noise $w_{r, i, k}$ :

$$
\tilde{r}_{i, k}=r_{i, k}+w_{r, i, k} .
$$

The variance of $w_{r, i, k}$ depends on the range, according to the following exponential model that has been derived from realworld round-trip-time UWB measurements in [28]:

$$
\sigma_{r, i, k}^{2}=\sigma_{0}^{2} \exp \left(\kappa_{\sigma} r_{i, k}\right),
$$

where $\sigma_{0}^{2}$ and $\kappa_{\sigma}$ are known constants.

Further, we denote the speed and orientation of the master by $V_{k}$ and $\phi_{k}$, respectively, whereas $\tilde{V}_{k}$ is the measurement of the speed and $\tilde{\phi}_{k}$ the measurement of the orientation. These measurements are expressed as

$$
\tilde{V}_{k}=V_{k}+w_{V, k}, \quad \tilde{\phi}_{k}=\phi_{k}+w_{\phi, k}
$$

where the noise terms $w_{V, k}$ and $w_{\phi, k}$ are zero-mean Gaussian random variables with known variances $\sigma_{V}^{2}$ and $\sigma_{\phi}^{2}$, respectively.

In this paper, assuming the measurement models described above, we propose a novel method to combine and improve existing estimators which are well known and commonly used. Specifically, we use a so called loosely-coupled approach, in which we fuse estimates already available from ranging measurements, with the Weighted Least Squares (WLS) method, and from velocity and orientation measurements, by performing dead-reckoning. Note that, in order to achieve the best possible accuracy, a tightly-coupled approach is necessary, where information from the ranging, velocity and orientation sensors are fused directly without preprocessing. However, the loosely-coupled approach is motivated by the reduced computational complexity as well as by the increased robustness and modularity that it offers.

This paper has two goals: First, to propose an highly accurate estimator of the position of the master by processing the results provided by existing ranging-only WLS and dead reckoning estimators. Second, to understand the fundamental limitations when estimating the position of the master by the fusion of information from these estimators. In the remainder of this section, we start by formulating the estimation problem that leads to the derivation of the proposed estimator.

The estimate of the master's position at time $k+1$ by using measurements available at that time is denoted by $\hat{\mathbf{x}}_{k+1 \mid k+1}=$ $\left[\begin{array}{ll}\hat{x}_{1, k+1 \mid k+1} & \hat{x}_{2, k+1 \mid k+1}\end{array}\right]^{T}$. We propose that it is derived as follows:

$$
\begin{aligned}
& \hat{x}_{1, k+1 \mid k+1}=\alpha_{1, k} \hat{x}_{1, k+1}^{(r)}+\beta_{1, k} \hat{x}_{1, k+1}^{(v)}, \\
& \hat{x}_{2, k+1 \mid k+1}=\alpha_{2, k} \hat{x}_{2, k+1}^{(r)}+\beta_{2, k} \hat{x}_{2, k+1}^{(v)},
\end{aligned}
$$

where $\hat{\mathbf{x}}_{k+1}^{(r)}=\left[\begin{array}{ll}\hat{x}_{1, k+1}^{(r)} & \hat{x}_{2, k+1}^{(r)}\end{array}\right]^{T}$ is the position estimate based only on the ranging measurements, and $\hat{\mathbf{x}}_{k+1}^{(v)}=$ $\left[\begin{array}{ll}\hat{x}_{1, k+1}^{(v)} & \hat{x}_{2, k+1}^{(v)}\end{array}\right]^{T}$ is the position estimate based on the dead reckoning block, which processes the position estimate at the previous time step and the on-board speed and orientation sensors. The terms $\alpha_{1, k}, \alpha_{2, k}, \beta_{1, k}$ and $\beta_{2, k}$ are the sensor fusion design parameters that need to be optimally chosen. 
Our estimation problem is separable on the $x_{1}$ and $x_{2}$ axes, in the sense that the estimates on the $x_{2}$ axis does not affect the $x_{1}$ axis, and vice versa. Therefore in the following we will provide derivations only for the $x_{1}$ component, because the derivations for the $x_{2}$ component are similar to those for the $x_{1}$ component.

Typically, the dead reckoning block provides us with an estimate having the following expression:

$$
\hat{x}_{1, k+1}^{(v)}=\hat{x}_{1, k \mid k}+\tilde{V}_{k} T \cos \tilde{\phi}_{k} .
$$

Note that we do not make the assumption of a linear motion model for the dead-reckoning block. Furthermore, the deadreckoning estimate is biased because the orientation measurement appears as the argument of a cosinus. It follows that the estimate (3) is biased. This motivates us to model our estimator (3) as

$$
\hat{x}_{1, k+1 \mid k+1} \triangleq x_{1, k+1}+w_{x_{1, k+1}},
$$

where $w_{x_{1, k+1}}$ is the error in the position estimate. This error has a non zero average, since the dead reckoning block gives a biased estimate.

It is possible to write a simplified form of Equations (3), by first noting that

$$
\begin{aligned}
\mathbb{E}\left\{\hat{x}_{1, k+1 \mid k+1}\right\}= & \alpha_{1, k} x_{1, k+1}+\beta_{1, k} x_{1, k+1} \\
& +\alpha_{1, k} \mathbb{E}\left\{w_{r, k+1}\right\} \\
& +\beta_{1, k} \mathbb{E}\left\{w_{v, k+1}\right\}+\beta_{1, k} \mathbb{E}\left\{w_{x, k}\right\} \\
= & x_{1, k+1}+\mathbb{E}\left\{w_{x, k+1}\right\}
\end{aligned}
$$

where $w_{r, k+1}$ and $w_{v, k+1}$ are the errors in the estimate obtained from the ranging block and from the dead-reckoning block, respectively. Now, in order to have $\alpha_{1, k} x_{1, k+1}+$ $\beta_{1, k} x_{1, k+1}=x_{1, k+1}$, it must be $\alpha_{1, k}+\beta_{1, k}=1$ and therefore

$$
\alpha_{1, k}=1-\beta_{1, k} \text {. }
$$

Hence, by substituting (7) in (3), we obtain

$$
\hat{x}_{1, k+1 \mid k+1}=\left(1-\beta_{1, k}\right) \hat{x}_{1, k+1}^{(r)}+\beta_{1, k} \hat{x}_{1, k+1}^{(v)},
$$

In the following, we will use the simplified expression (8) instead of (3).

From (6) it follows that the error is

$$
\begin{aligned}
w_{x_{1, k+1}}= & \hat{x}_{1, k+1 \mid k+1}-x_{1, k+1} \\
= & \left(1-\beta_{1, k}\right) w_{x_{1, k+1}}^{(r)}+\beta_{1, k} w_{x_{1, k}} \\
& +\beta_{1, k} T \tilde{V}_{k} \cos \tilde{\phi}_{k}-\beta_{1, k} T V_{k} \cos \phi_{k},
\end{aligned}
$$

where $w_{x_{1, k+1}}^{(r)}$ is the error of the estimates obtained from the ranging. To develop an estimator for the master's position, we define a cost function that takes into account the estimator variance and bias simultaneously. We define the bias term of the estimation error as $\mu_{w 1, k+1} \triangleq$ $\mathbb{E}\left\{w_{x_{1, k+1}}\right\}$, and the variance term of the estimation error as $\sigma_{w 1, k+1}^{2} \triangleq \mathbb{E}\left\{\left(\hat{x}_{1, k+1 \mid k+1}-\mathbb{E}\left\{\hat{x}_{1, k+1 \mid k+1}\right\}\right)^{2}\right\}=$ $\mathbb{E}\left\{\left(w_{x_{1, k+1}}-\mathbb{E}\left\{w_{x_{1, k+1}}\right\}\right)^{2}\right\}$.

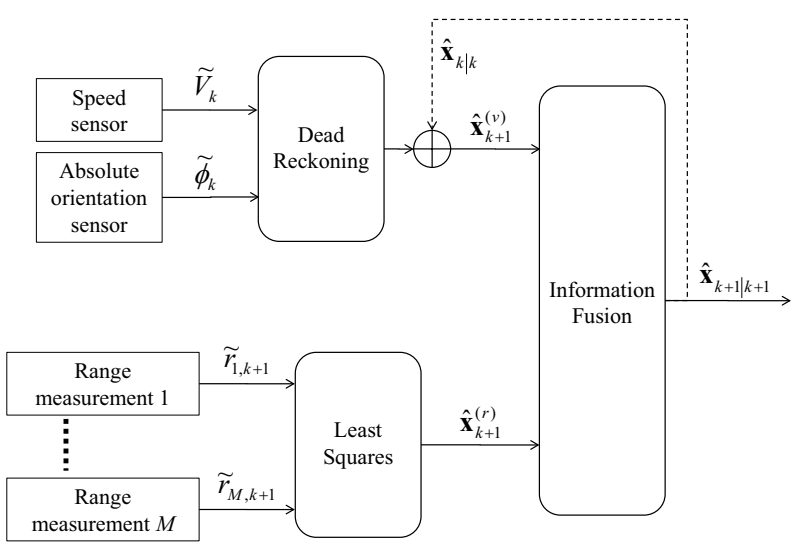

Fig. 2. Sensor fusion system architecture used at a master node for estimating its position at time $k+1$ given available information. The dead reckoning block gives speed and absolute orientation information, whereas the least square block gives ranging information as computed with respect to slave nodes. The information provided by these two blocks is then fused to provide an accurate estimation $\hat{\mathbf{x}}_{k+1 \mid k+1}$

We are now ready to formulate a Pareto optimization problem to select the fusion coefficients at each time $k$ :

$$
\begin{array}{ll}
\min _{\beta_{1, k}} & \rho_{1, k} \mu_{w 1, k}^{2}+\left(1-\rho_{1, k}\right) \sigma_{w 1, k}^{2} \\
\text { s.t. } & \beta_{1, k} \in \mathscr{B}=[-1,1], \quad \rho_{1, k} \in \mathscr{R}=[0,1] .
\end{array}
$$

This problem is motivated by that we would like to reduce as much as possible both the average of the estimation error, namely the bias due to the dead reckoning block, and the variance of the estimation error. The Pareto weighting factor (or scalarization coefficient) $\rho_{1, k}$ must be chosen for each time $k$ so to trade off the low bias and high variance of the error of the ranging estimate with the high bias and low variance of the error of the dead-reckoning estimate [37, Section 4.7.5]. Based on the statistical properties of the bias, which we characterize below, the bias itself may grow unstable with time, which motivates the constraint $\beta_{1, k} \in \mathscr{B}$. The intuition is that the minimization of both the average and the variance of the estimation error at every time $k$ does not ensure that the bias is stable or decreases over time. Notice that in the special case of $\rho_{1, k}=0 \forall k$, the cost function is the variance of the estimation error, and when $\rho_{1, k}=0.5 \forall k$ the cost function is the mean square error (MSE).

The challenge for such an optimization is the analytical characterization of the cost function (11), which we study in the following section.

\section{DERIVATION OF A SENSOR FUSION Estimator}

The architecture of the proposed estimator is shown in Fig. 2. In the following subsections, we provide a statistical characterization of the ranging estimate $\hat{\mathbf{x}}_{k+1}^{(r)}$ and the deadreckoning estimate $\hat{\mathbf{x}}_{k+1}^{(v)}$. Then, in subsection III-D we solve the optimization problem, thus achieving the optimal weighting coefficients $\beta_{1, k}$ and $\beta_{2, k}$ to use in Eq. (8). The original contribution of this section is the statistical characterization of the estimation errors, and the optimal weighting derivation in Proposition 3.7. 


\section{A. Ranging estimate statistical characterization}

In this subsection we give a new statistical characterization of the estimation error of the ranging estimate. We consider the problem of finding the first and second order moments of the ranging estimates of the $x_{1}$ and $x_{2}$ components of the position. This requires first a derivation of the ranging estimates. In the following, for the sake of simple notation, we drop the $k+1$ indices.

Since it must be that $M \geq 4$ for linear-based ranging estimates, the master's position estimate $\hat{\mathbf{x}}^{(r)}$ may be obtained by following a commonly employed strategy (see, e.g., [38]) as the solution of $\mathbf{A} \hat{\mathbf{x}}^{(r)}=\tilde{\mathbf{s}}$, where $\mathbf{A}$ and $\tilde{\mathbf{s}}$ are easily seen to be

$$
\begin{gathered}
\mathbf{A}=\left[\begin{array}{cc}
2\left(s_{1,1}-s_{M, 1}\right) & 2\left(s_{1,2}-s_{M, 2}\right) \\
\vdots & \vdots \\
2\left(s_{M-1,1}-s_{M, 1}\right) & 2\left(s_{M-1,2}-s_{M, 2}\right)
\end{array}\right], \\
\tilde{\mathbf{s}}=\left[r_{M}^{2}-r_{1}^{2}, \ldots, r_{M}^{2}-r_{M-1}^{2}\right]^{T}+\mathbf{a}+\mathbf{w}_{\mathbf{s}},
\end{gathered}
$$

where the constant vector $\mathbf{a}$ is given by the following function of the known coordinates of the slaves

$$
\mathbf{a}=\left[\begin{array}{c}
s_{1,1}^{2}-s_{M, 1}^{2}+s_{1,2}^{2}-s_{M, 2}^{2} \\
\cdots \\
s_{M-1,1}^{2}-s_{M, 1}^{2}+s_{M-1,2}^{2}-s_{M, 2}^{2}
\end{array}\right],
$$

and $\mathbf{w}_{\mathbf{s}}$ is a random vector given by

$$
\mathbf{w}_{\mathbf{s}} \triangleq\left[\begin{array}{c}
w_{r_{M}}^{2}+2 r_{M} w_{r_{M}}-w_{r_{1}}^{2}-2 r_{1} w_{r_{1}} \\
\ldots \\
w_{r_{M}}^{2}+2 r_{M} w_{r_{M}}-w_{r_{M-1}}^{2}-2 r_{M-1} w_{r_{M-1}}
\end{array}\right] .
$$

We derive the ranging estimate by the weighted least squares (WLS) technique, since ranging measurements are affected by unequal statistical errors across the various links. Then, the solution to the following classical WLS optimization problem gives the ranging estimates: $\min _{\hat{\mathbf{x}}^{(r)}}\left\|\mathbf{W}^{\frac{1}{2}}\left(\tilde{\mathbf{s}}-\mathbf{A} \hat{\mathbf{x}}^{(r)}\right)\right\|$, where $\mathbf{W}$ is a positive-definite weighting matrix introduced for the purpose of emphasizing the contribution of those range measurements that are deemed to be more reliable [39], and $\|\cdot\|$ is the Euclidean norm. A common choice for $\mathbf{W}$ is $\mathbf{R}^{-1}$, namely the inverse of the noise covariance matrix:

$$
\mathbf{R} \triangleq \mathbb{E}\left\{\left(\mathbf{w}_{s}-\mathbb{E}\left\{\mathbf{w}_{s}\right\}\right)\left(\mathbf{w}_{s}-\mathbb{E}\left\{\mathbf{w}_{s}\right\}\right)^{T}\right\} .
$$

Such a choice is motivated by that it would give a maximum likelihood estimator in the case of Gaussian errors. Thus the well-known solution to the WLS problem is

$$
\hat{\mathbf{x}}^{(r)}=\left(\mathbf{A}^{T} \mathbf{W A}\right)^{-1} \mathbf{A}^{T} \mathbf{W} \tilde{\mathbf{s}},
$$

where $\mathbf{W}=\mathbf{R}^{-1}$.

We are now ready to introduce the new part of this subsection. From (16) we can characterize the first and second order moments of the error in the ranging estimate. First, however, we need the expression of the inverse of the error covariance matrix. This inverse matrix has a simple expression and is provided by the following lemma:
Lemma 3.1: Consider the matrix $\mathbf{R}$ as defined in (15). The matrix has full rank, and the inverse is

$$
\mathbf{R}^{-1}=\left(\mathbf{I}-\frac{1}{1+q} \mathbf{G} \mathbf{1 1}{ }^{T}\right) \mathbf{D}^{-1}
$$

where $\mathbf{I}$ is the $(M-1) \times(M-1)$ identity matrix, $\mathbf{D}$ is a $(M-1) \times(M-1)$ diagonal matrix whose entries are $\mathbf{D}_{l l}=$ $4 r_{l}^{2} \sigma_{w_{r_{l}}}^{2}+2 \sigma_{w_{r_{l}}}^{4}, l=1, \ldots, M-1, \mathbf{G}=p \mathbf{D}^{-1}$ is a $(M-1) \times$ $(M-1)$ matrix with $p=4 r_{M}^{2} \sigma_{w_{r_{M}}}^{2}+2 \sigma_{w_{r_{M}}}^{4}, q=\sum_{i=1}^{M-1} p / \mathbf{D}_{i i}$, and $\mathbf{1}$ is the all ones vector.

Proof: The lemma follows from tedious algebraic computations, the matrix inversion lemma, and the Woodbury identity [39].

Based on Eq. (16) and the previous lemma, we are now in the position of deriving the expectation and the variance of the ranging estimation error $\mathbf{w}_{r}$, defined as $\mathbf{w}_{r} \triangleq \hat{\mathbf{x}}^{(r)}-\mathbf{x}=$ $\left[\begin{array}{cc}w_{x_{1}}^{(r)} & w_{x_{2}}^{(r)}\end{array}\right]^{T}$.

Lemma 3.2: The expectation of the ranging estimation error is given by:

$$
\begin{aligned}
\mathbb{E}\left\{\mathbf{w}_{r}\right\}= & \left(\mathbf{A}^{T} \mathbf{W} \mathbf{A}\right)^{-1} \mathbf{A}^{T} \mathbf{W} \\
& \times\left(\mathbf{1} \sigma_{w_{r_{M}}}^{2}-\left[\sigma_{w_{r_{1}}}^{2}, \ldots, \sigma_{w_{r_{M}-1}}^{2}\right]^{T}\right) .
\end{aligned}
$$

Proof: See Appendix A.

Furthermore, we have the following result:

Lemma 3.3: The correlation of the range estimation error is given by

$$
\mathbb{E}\left\{\mathbf{w}_{r}^{2}\right\}=\left(\mathbf{A}^{T} \mathbf{W A}\right)^{-1} \mathbf{A}^{T} \mathbf{W} \mathbf{C} \mathbf{W}^{T} \mathbf{A}\left(\mathbf{A}^{T} \mathbf{W A}\right)^{-1},
$$

where $\mathbf{C}$ is a matrix whose diagonal elements are $\mathbf{C}_{l l}=$ $3 \sigma_{w_{r_{M}}}^{4}+4 r_{M}^{2} \sigma_{w_{r_{M}}}^{2}+3 \sigma_{w_{r_{l}}}^{4}+4 r_{l}^{2} \sigma_{w_{r_{l}}}^{2}-2 \sigma_{w_{r_{M}}}^{2} \sigma_{w_{r_{l}}}^{2}$, with $l=1, \ldots, M$, and the off-diagonal elements are $\mathbf{C}_{l j}=$ $3 \sigma_{w_{r_{M}}}^{4}-\sigma_{w_{r_{M}}}^{2} \sigma_{w_{r_{j}}}^{2}+4 r_{M}^{2} \sigma_{w_{r_{M}}}^{2}-\sigma_{w_{r_{l}}}^{2} \sigma_{w_{r_{M}}}^{2}+\sigma_{w_{r_{l}}}^{2} \sigma_{w_{r_{j}}}^{2}$, with $l \neq j$.

Proof: See Appendix B.

This concludes the efforts to characterize the statistics of the ranging estimation error. In the following subsection, we focus on the statistics of the dead-reckoning estimation.

\section{B. Dead-reckoning estimate statistical characterization}

In this subsection, we give a new characterization of the estimation errors of the dead-reckoning block. We have the following results for the first and second order moments of the dead-reckoning estimate:

Lemma 3.4: Let $\tilde{\phi}(k)$ be Gaussian, and assume that $\tilde{V}(k)$ and $\tilde{\phi}(k)$ are statistically independent. Then

$$
\begin{aligned}
\mathbb{E}\left\{\tilde{V}_{k} \cos \left(\tilde{\phi}_{k}\right)\right\} & =V_{k} \cos \left(\phi_{k}\right) e^{-\frac{\sigma_{\phi}^{2}}{2}}, \\
\mathbb{E}\left\{\tilde{V}_{k}^{2} \cos ^{2}\left(\tilde{\phi}_{k}\right)\right\} & =\sigma_{V}^{2}\left(\frac{1}{2}+\frac{1}{2} \cos \left(2 \phi_{k}\right) e^{-2 \sigma_{\phi}^{2}}\right) .
\end{aligned}
$$

Proof: See Appendix C.

In the following subsection, we use these results to characterize the estimation bias. 


\section{Estimation Bias}

We can now put together the statistical characterization of the ranging and dead-reckoning estimations. We have the following results that give the terms $\mu_{w 1, k}$ and $\sigma_{w 1, k}^{2}$ in Eq. (11):

Lemma 3.5: Consider the estimation error (9). The bias is

$$
\begin{aligned}
& \mu_{w 1, k+1}=\mathbb{E}\left\{w_{x_{1, k+1}}\right\}=\left(1-\beta_{1, k}\right) \mathbb{E}\left\{w_{x_{1, k+1}}^{(r)}\right\} \\
& +\beta_{1, k} \mathbb{E}\left\{w_{x_{1, k}}\right\}+\beta_{1, k} T V_{k} \cos \left(\phi_{k}\right)\left(e^{-\frac{\sigma_{\phi}^{2}}{2}}-1\right) .
\end{aligned}
$$

\section{Proof: See Appendix D.}

Remark From the previous lemma, we see that the average of the estimation error at time $k+1$ depends on the bias of time $k$. To avoid an accumulation of the bias, we need to impose a condition on $\beta_{1, k}$. Thus, the absolute value of the average of the estimation error must be non-expansive, which can be easily achieved when $\left|\beta_{1, k}\right| \in[0,1]$, and thus we have $\mathscr{B}=[-1,1]$ in the Pareto optimization problem (11).

Lemma 3.6: Consider the estimation error (9). Then, the second order moment is

$$
\mathbb{E}\left\{w_{x_{1, k+1}}^{2}\right\}=\beta_{1, k}^{2} a_{k}+2 \beta_{1, k} b_{k}+\mathbb{E}\left\{\left(w_{x_{1, k+1}}^{(r)}\right)^{2}\right\}
$$

where

$$
\begin{aligned}
& a_{k}=\mathbb{E}\left\{\left(w_{x_{1, k+1}}^{(r)}\right)^{2}\right\}+\mathbb{E}\left\{w_{x_{1, k}}^{2}\right\}+T^{2} \mathbb{E}\left\{\tilde{V}_{k}^{2} \cos ^{2}\left(\tilde{\phi}_{k}\right)\right\} \\
& +T^{2} V_{k}^{2} \cos ^{2}\left(\phi_{k}\right)\left(1-2 e^{-\frac{\sigma_{\phi}^{2}}{2}}\right)-2 \mathbb{E}\left\{w_{x_{1, k}}\right\} \mathbb{E}\left\{w_{x_{1, k+1}}^{(r)}\right\} \\
& -2 \mathbb{E}\left\{w_{x_{1, k+1}}^{(r)}\right\} T V_{k} \cos \left(\phi_{k}\right)\left(e^{-\frac{\sigma_{\phi}^{2}}{2}}-1\right) \\
& +2 \mathbb{E}\left\{w_{x_{1, k}}\right\} T V_{k} \cos \left(\phi_{k}\right)\left(e^{-\frac{\sigma_{\phi}^{2}}{2}}-1\right) \\
& b_{k}=-\mathbb{E}\left\{\left(w_{x_{1, k+1}}^{(r)}\right)^{2}\right\}+\mathbb{E}\left\{w_{x_{1, k}}\right\} \mathbb{E}\left\{w_{x_{1, k+1}}^{(r)}\right\} \\
& +\mathbb{E}\left\{w_{x_{1, k+1}}^{(r)}\right\} T V_{k} \cos \left(\phi_{k}\right)\left(e^{-\frac{\sigma_{\phi}^{2}}{2}}-1\right) .
\end{aligned}
$$

Proof: The result follows from Lemma 3.2, Eq. (17), and Proposition 3.4.

Finally, to derive an expression for the variance of the estimation error $\sigma_{w 1, k}^{2}$ that we need in (11), we define the variables $v_{r}, v_{x_{1}}$ and $v_{v}$ as

$$
\begin{aligned}
& v_{r} \triangleq w_{x_{1, k+1}}^{(r)}-\mathbb{E}\left\{w_{x_{1, k+1}}^{(r)}\right\}, v_{x_{1}} \triangleq w_{x_{1, k}}-\mathbb{E}\left\{w_{x_{1, k}}\right\} \\
& v_{v} \triangleq T \tilde{V}_{k} \cos \left(\tilde{\phi}_{k}\right)-T V_{k} \cos \left(\phi_{k}\right) e^{-\frac{\sigma_{\phi}^{2}}{2}}
\end{aligned}
$$

Notice that $v_{r}, v_{x_{1}}$ and $v_{v}$ are independent; we denote their variances as $\sigma_{v_{r}}^{2}, \sigma_{v_{x_{1}}}^{2}$ and $\sigma_{v_{v}}^{2}$ respectively. Also, note that $\mathbb{E}\left\{v_{r}\right\}=\mathbb{E}\left\{v_{x_{1}}\right\} \stackrel{x_{1}}{=}$. By substituting the expression for the error bias (20) in Eq. (21) we obtain

$$
\begin{aligned}
\sigma_{w 1, k+1}^{2}= & \mathbb{E}\left\{\left(\left(1-\beta_{1, k}\right) w_{x_{1, k+1}}^{(r)}+\beta_{1, k} w_{x_{1, k}}\right.\right. \\
& +\beta_{1, k} T \tilde{V}_{k} \cos \left(\tilde{\phi}_{k}\right)-\beta_{1, k} T V_{k} \cos \left(\phi_{k}\right) \\
& -\left(1-\beta_{1, k}\right) \mathbb{E}\left\{w_{x_{1, k+1}}^{(r)}\right\}-\beta_{1, k} \mathbb{E}\left\{w_{x_{1, k}}\right\} \\
& \left.\left.+\beta_{1, k} T V_{k} \cos \left(\phi_{k}\right) e^{-\frac{\sigma_{\phi}^{2}}{2}}\right)^{2}\right\} \\
= & \mathbb{E}\left\{\left(1-\beta_{1, k}\right)^{2} v_{r}^{2}+\beta_{1, k}^{2} v_{x_{1}}^{2}+\beta_{1, k}^{2} v_{v}^{2}\right. \\
& +2\left(1-\beta_{1, k}\right) \beta_{1, k} v_{r} v_{x_{1}}+2\left(1-\beta_{1, k}\right) \beta_{1, k} v_{r} v_{v} \\
& \left.+2 \beta_{1, k}^{2} v_{x_{1}} v_{v}\right\} \\
= & \left(1-\beta_{1, k}\right)^{2} \sigma_{r}^{2}+\beta_{1, k}^{2} \sigma_{x}^{2}+\beta_{1, k}^{2} \sigma_{v}^{2}
\end{aligned}
$$

where we have used that $v_{r}, v_{x_{1}}$ and $v_{v}$ are independent and $\mathbb{E}\left\{v_{r}\right\}=\mathbb{E}\left\{v_{x_{1}}\right\}=0$

\section{Pareto Optimization}

In this section, we put together the results of the previous sections to derive a position estimator by solving the optimization problem in (11). Lemmata 3.5 and 3.6 give us the analytical expression of the cost function of problem (11). Thus, we have the following result, which is one of the core contributions of this paper:

Proposition 3.7: Consider optimization problem (11). Let the mean and the variance of the estimation error (9) be computed by Lemmata 3.5 and 3.6. Then, the optimal solution for a fixed Pareto weighting factor $\rho_{1, k}$ is

$$
\beta_{1, k}^{*}\left(\rho_{1, k}\right)=\max (-1, \min (\xi, 1)),
$$

with

$$
\xi=\frac{2\left(1-\rho_{1, k}\right) \sigma_{v_{r}}^{2}-2 \rho_{1, k} \gamma \mathbb{E}\left\{w_{r, k+1}\right\}}{2\left(1-\rho_{1, k}\right) \eta+2 \rho_{1, k} \gamma^{2}},
$$

where

$\eta \triangleq \sigma_{v_{r}}^{2}+\sigma_{v_{x}}^{2}+\sigma_{v_{v}}^{2}$,

$\gamma \triangleq-\mathbb{E}\left\{w_{r, k+1}\right\}+\mathbb{E}\left\{w_{x, k}\right\}+T V_{k} \cos \left(\phi_{k}\right)\left(e^{-\frac{\sigma_{\phi}^{2}}{2}}-1\right)$.

Proof: See Appendix E.

As a particular case of the previous proposition is given by the MSE, when the optimal solution of problem (11) is obtained with the Pareto weighting factor $\rho_{1, k}=0.5 \forall k$ and results

$$
\beta_{1, k}^{*}=\max \left(-1, \min \left(-\frac{b_{k}}{a_{k}}, 1\right)\right),
$$

where $a_{k}$ and $b_{k}$ are given by Eq. (22) and Eq. (23), respectively. In general, the optimal value of $\beta_{1, k}$ in (25) depends on the scalarization coefficient $\rho_{1, k}$ (see [37, Section 4.7]). The best value of such a coefficient is found by building the Pareto trade-off curve and selecting the "knee-point" on this curve [37]. Thus, we choose $\rho_{1, k}^{*}$ such that $\mu_{w 1, k}$ and $\sigma_{w 1, k}^{2}$ computed in $\beta_{1, k}^{*}\left(\rho_{1, k}^{*}\right)$ are $\sigma_{w 1, k}^{2} \simeq \mu_{w 1, k}^{2}$. This is given by the solution to the following further optimization problem

$$
\min _{\rho_{1, k}}\left(\sigma_{w 1, k}^{2}\left(\beta_{1, k}^{*}\left(\rho_{1, k}\right)\right)-\mu_{w 1, k}^{2}\left(\beta_{1, k}^{*}\left(\rho_{1, k}\right)\right)\right)^{2},
$$


where we have evidenced that $\mu_{w 1, k}$ and $\sigma_{w 1, k}^{2}$ are computed in $\beta_{1, k}^{*}\left(\rho_{1, k}\right)$, according to Eqs. (20) and (24). This problem is highly non-linear, but simple and standard numerical procedures, based on a discrimination of $\rho_{1, k}$, can be employed to compute approximately the optimal Pareto coefficient $\rho_{1, k}^{*}$. The standard numerical procedure is described in [37, Section 4.7.5]. Such a procedure consists of first defining a set of values that $\rho_{1, k}$ can assume, then for each such value the procedure does the following steps:

1) compute $\beta^{*}\left(\rho_{1, k}\right)$ via (25) for the current value of $\rho_{1, k}$,

2) use the value of $\beta^{*}\left(\rho_{1, k}\right)$ computed in 1) and plug it in (20) and in (24) to evaluate $\mu_{w 1, k}^{2}\left(\beta_{1, k}^{*}\left(\rho_{1, k}\right)\right)$ and $\sigma_{w 1, k}^{2}\left(\beta_{1, k}^{*}\left(\rho_{1, k}\right)\right)$;

$3)$ use the values $\mu_{w 1, k}^{2}\left(\beta_{1, k}^{*}\left(\rho_{1, k}\right)\right) \quad$ and $\sigma_{w 1, k}^{2}\left(\beta_{1, k}^{*}\left(\rho_{1, k}\right)\right)$ computed at step 2) to compute

$$
\left(\sigma_{w 1, k}^{2}\left(\beta_{1, k}^{*}\left(\rho_{1, k}\right)\right)-\mu_{w 1, k}^{2}\left(\beta_{1, k}^{*}\left(\rho_{1, k}\right)\right)\right)^{2},
$$

and store such a value.

The approximate optimum $\rho_{1, k}^{*}$ is the one which corresponds to the minimum of the stored values of (28). Such a $\rho_{1, k}$ corresponds to the "knee point" of the Pareto trade off curve [37, Section 4.7.5].

Finally, recall that the values of the optimal $\beta_{2, k}$ and $\rho_{x_{2, k}}$ yielding the $x_{2}$ component of the position estimate are obtained similarly to what done for the $x_{1}$ component.

\section{Cramér-Rao Lower Bound}

In this section, we are interested in investigating the fundamental performance limitation of our estimator proposed in the previous section. To do so, given any estimation problem, the CRLB is a fundamental tool for performance analysis, since it quantifies the best achievable mean-square error performance of estimators [39]. We wish to derive such a bound to compare it to the performance of our estimator. There are two fundamental approaches: the parametric CRLB and posterior CRLB.

The parametric CRLB, that we denote as ParCRLB, is the CRLB evaluated for a given state-space trajectory [19]. Therefore it is equivalent to the problem of estimating unknown deterministic states considering a zero process noise in the dynamical system model; for the proof see, e.g., the derivations in [40]. In other words, the state is treated as a deterministic but unknown parameter for the purpose of the evaluation of the ParCRLB. Therefore, the ParCRLB is simple to evaluate in a numerical simulation environment where the true trajectory is known, because the equations required for its calculation do not contain any statistical expectation operator; see, e. g., [41]. However, due to its nature, the ParCRLB does not provide information about the fundamental performance limitations in the general case. For such a case, we need to investigate the posterior CRLB, which we do in the following.

\section{A. Posterior CRLB}

For recursive estimation problems in the Bayesian framework, where the state is treated as a random variable, the bound is denoted as the posterior CRLB (PCRLB). A recursive formulation of the PCRLB has been first introduced in [32]. In the following, we derive such a bound for our localization problem. The core contribution of this section is summarized in Proposition 4.5 below.

Differently from the case of the ParCRLB, where one is interested in calculating the performance bound for a given trajectory, here we aim at characterizing the fundamental performance limits, on the average, for all the trajectories belonging to a class, which is defined by a dynamical model. Therefore we introduce the state $\mathbf{p}_{k} \in \mathbb{R}^{4}$ to model the bidimensional position of the master, its absolute speed and its orientation, that is $\mathbf{p}_{k}=\left[\begin{array}{llll}x_{1, k} & x_{2, k} & V_{k} & \phi_{k}\end{array}\right]^{T}$, and we consider the following non-linear state-space model:

$$
\begin{aligned}
\mathbf{p}_{k+1} & =\mathbf{f}\left(\mathbf{p}_{k}\right)+\mathbf{v}_{k} \\
\mathbf{y}_{k} & =\mathbf{h}\left(\mathbf{p}_{k}\right)+\mathbf{e}_{k},
\end{aligned}
$$

where $\quad \mathbf{v}_{k}=\left[\begin{array}{llll}v_{1, k} & v_{2, k} & v_{3, k} & v_{4, k}\end{array}\right]^{T} \in \mathbb{R}^{4} \sim$ $\mathcal{N}(0, \mathbf{Q})$ is the white Gaussian process noise with $\mathbf{Q}=$ $\operatorname{diag}\left(\sigma_{1}^{2}, \sigma_{2}^{2}, \sigma_{3}^{2}, \sigma_{4}^{2}\right)$, where it is natural to assume that $\sigma_{1}=\sigma_{2}$, namely that the process noise on the two coordinates has same variance, and $\mathbf{e}_{k} \in \mathbb{R}^{M+2} \sim \mathcal{N}\left(0, \mathbf{R}_{\mathbf{k}}\right)$ is the white Gaussian measurement noise, which is independent of $\mathbf{v}_{k}$, with $\mathbf{R}_{\mathbf{k}}=\operatorname{diag}\left(\sigma_{r, 1, k}^{2}, \ldots, \sigma_{r, M, k}^{2}, \sigma_{V}^{2}, \sigma_{\phi}^{2}\right)$. Simple algebra gives that the state update and measurement functions are, respectively,

$$
\begin{aligned}
\mathbf{f}\left(\mathbf{p}_{k}\right)= & {\left[\begin{array}{c}
x_{1, k}+T V_{k} \cos \phi_{k} \\
x_{2, k}+T V_{k} \sin \phi_{k} \\
V_{k} \\
\phi_{k}
\end{array}\right], } \\
\mathbf{h}\left(\mathbf{p}_{k}\right)= & {\left[\begin{array}{c}
\left.h_{1}, \ldots, h_{M}, h_{M+1}, h_{M+2}\right]^{T} \\
\sqrt{\left(s_{1,1}-x_{1, k}\right)^{2}+\left(s_{1,2}-x_{2, k}\right)^{2}} \\
\vdots \\
\sqrt{\left(s_{M, 1}-x_{1, k}\right)^{2}+\left(s_{M, 2}-x_{2, k}\right)^{2}} \\
V_{k} \\
\phi_{k}
\end{array}\right], }
\end{aligned}
$$

where we recall that $T$ is the sampling interval.

Consider the dynamical system of (29) and (30), and denote as $\mathbf{P}_{k}$ the covariance matrix of any unbiased estimator of the system's state at time $k$. Then, $\mathbf{P}_{k}$ is lower bounded by the inverse of the Fisher information matrix $\mathbf{J}_{k}$, that is $\mathbf{J}_{k}^{-1} \preceq \mathbf{P}_{k}$. Such a matrix can be recursively computed as [32]

$$
\mathbf{J}_{k+1}=\mathbf{D}_{k}^{22}-\mathbf{D}_{k}^{21}\left(\mathbf{J}_{k}+\mathbf{D}_{k}^{11}\right)^{-1} \mathbf{D}_{k}^{12}
$$

with

$$
\begin{aligned}
\mathbf{D}_{k}^{11}= & -\mathbb{E}\left\{\nabla_{\mathbf{x}_{k}}\left[\nabla_{\mathbf{x}_{k}} \log p\left(\mathbf{x}_{k+1} \mid \mathbf{x}_{k}\right)\right]^{T}\right\} \\
\mathbf{D}_{k}^{21}= & \left(\mathbf{D}_{k}^{12}\right)^{T}=-\mathbb{E}\left\{\nabla_{\mathbf{x}_{k}}\left[\nabla_{\mathbf{x}_{k+1}} \log p\left(\mathbf{x}_{k+1} \mid \mathbf{x}_{k}\right)\right]^{T}\right\} \\
\mathbf{D}_{k}^{22}= & -\mathbb{E}\left\{\nabla_{\mathbf{x}_{k+1}}\left[\nabla_{\mathbf{x}_{k+1}} \log p\left(\mathbf{x}_{k+1} \mid \mathbf{x}_{k}\right)\right]^{T}\right\} \\
& -\mathbb{E}\left\{\nabla_{\mathbf{x}_{k+1}}\left[\nabla_{\mathbf{x}_{k+1}} \log p\left(\mathbf{y}_{k+1} \mid \mathbf{x}_{k}\right)\right]^{T}\right\}
\end{aligned}
$$

where the initial condition is given by the information matrix $\mathbf{J}_{0}$, which is computed based on the initial prior density $p\left(\mathbf{x}_{0}\right)$. 
The system model in (29) and (30) has additive Gaussian noise. Hence, the terms in (32), (33) and (34) may be expressed as (see [32, eq. (34) - (36)])

$$
\begin{aligned}
& \mathbf{D}_{k}^{11}=\mathbb{E}\left\{\left[\nabla_{\mathbf{p}_{k}} \mathbf{f}\left(\mathbf{p}_{k}\right)\right] \mathbf{Q}^{-1}\left[\nabla_{\mathbf{p}_{k}} \mathbf{f}\left(\mathbf{p}_{k}\right)\right]^{T}\right\} \\
& \mathbf{D}_{k}^{12}=-\mathbb{E}\left\{\left[\nabla_{\mathbf{p}_{k}} \mathbf{f}\left(\mathbf{p}_{k}\right)\right]\right\} \mathbf{Q}^{-1}, \\
& \mathbf{D}_{k}^{22}=\mathbf{Q}^{-1}+\mathbb{E}\left\{\left[\nabla_{\mathbf{p}_{k}} \mathbf{h}\left(\mathbf{p}_{k}\right)\right] \mathbf{R}_{\mathbf{k}}{ }^{-1}\left[\nabla_{\mathbf{p}_{k}} \mathbf{h}\left(\mathbf{p}_{k}\right)\right]^{T}\right\} .
\end{aligned}
$$

In general, there is no guarantee that the expectations in (35)(37) exist. In other words, the CRLB may not exist. Therefore, in the following we establish the existence of the CRLB by deriving closed form expressions for the first two terms, namely $\mathbf{D}_{k}^{11}$ and $\mathbf{D}_{k}^{12}$, while we derive bounds for the third term $\mathbf{D}_{k}^{22}$. We start by proving the following useful Lemma:

Lemma 4.1: Consider the dynamical system in (29) - (30), denote the speed and the orientation at time $k=0$ as $V_{0}$ and $\phi_{0}$, respectively, and define the following quantities: $c_{0} \triangleq \cos \phi_{0}, \quad s_{0} \triangleq \sin \phi_{0}, \quad c_{0}^{\prime} \triangleq \cos 2 \phi_{0}, \quad s_{0}^{\prime} \triangleq$ $\sin 2 \phi_{0}, \quad \varepsilon_{k} \triangleq e^{-\frac{(k-1) \sigma_{4}^{2}}{2}}$. Then

$$
\begin{aligned}
\mathbb{E}\left\{V_{k}\right\} & =V_{0}, & \mathbb{E}\left\{V_{k}^{2}\right\}=(k-1) \sigma_{3}^{2}+V_{0}^{2} \\
\mathbb{E}\left\{\cos \phi_{k}\right\} & =c_{0} \varepsilon_{k}, & \mathbb{E}\left\{\sin \phi_{k}\right\}=s_{0} \varepsilon_{k} \\
\mathbb{E}\left\{\sin \phi_{k} \cos \phi_{k}\right\} & =\frac{1}{2} s_{0}^{\prime} \varepsilon_{k}^{2}, & \mathbb{E}\left\{\cos ^{2} \phi_{k}\right\}=\frac{1}{2}+\frac{1}{2} c_{0}^{\prime} \varepsilon_{k}^{4}
\end{aligned}
$$

Proof: See Appendix F.

Then, we note that the gradient of $\mathbf{f}$ is easily evaluated as

$$
\nabla_{\mathbf{p}_{k}} \mathbf{f}\left(\mathbf{p}_{k}\right)=\left[\begin{array}{cccc}
1 & 0 & 0 & 0 \\
0 & 1 & 0 & 0 \\
T \cos \phi_{k} & T \sin \phi_{k} & 1 & 0 \\
-T V_{k} \sin \phi_{k} & T V_{k} \cos \phi_{k} & 0 & 1
\end{array}\right] .
$$

By substituting Eq. (38) in Eq.(35) and using Lemma 4.1 we obtain Eq. (39) (see top of next page). Note that, when $k$ tends to infinity, this matrix tends to a diagonal matrix, because $\varepsilon_{k}$ tends to zero, but the right-lower element tends to infinity. This implies that, as $k$ grows, the most relevant contribution to the Fisher information matrix in Eq. (31) is given by the $\mathbf{D}_{k}^{22}$ term. Therefore, in the limit case, only the measurements influence the PCRLB, not the model.

Similarly, by substituting Eq. (38) in Eq.(36), we obtain:

$$
\mathbf{D}_{k}^{12}=-\left[\begin{array}{cccc}
\sigma_{1}^{-2} & 0 & 0 & 0 \\
0 & \sigma_{2}^{-2} & 0 & 0 \\
T \sigma_{1}^{-2} c_{0} \varepsilon_{k} & T \sigma_{2}^{-2} s_{0} \varepsilon_{k} & \sigma_{3}^{-2} & 0 \\
-T \sigma_{1}^{-2} V_{0} s_{0} \varepsilon_{k} & T \sigma_{2}^{-2} V_{0} c_{0} \varepsilon_{k} & 0 & \sigma_{4}^{-2}
\end{array}\right] .
$$

We now turn our attention to the $\mathbf{D}_{k}^{22}$ term in (31). For the sake of simple notation, we drop the $k$ subscript in the remainder of this section and we define the following quantity:

$$
d_{i, j} \triangleq \frac{x_{i}-s_{j, i}}{\sqrt{\left(x_{1}-s_{j, 1}\right)^{2}+\left(x_{2}-s_{j, 2}\right)^{2}}} .
$$

After some algebra, the gradient of $\mathbf{h}$ is

$$
\nabla_{\mathbf{p}} \mathbf{h}(\mathbf{p}) \triangleq\left[\begin{array}{ccccc}
d_{1,1} & \ldots & d_{1, M} & 0 & 0 \\
d_{2,1} & \ldots & d_{2, M} & 0 & 0 \\
0 & \ldots & 0 & 1 & 0 \\
0 & \ldots & 0 & 0 & 1
\end{array}\right]
$$

Let us introduce the following definition for the matrix $\Pi$ in Eq. (40) (see top of next page). By substituting Eq. (41) in Eq. (37), and using the above definitions, we obtain

$$
\mathbf{D}^{22}=\mathbf{Q}^{-1}+\left[\begin{array}{cc}
\boldsymbol{\Pi} & \mathbf{0} \\
\mathbf{0} & \mathbf{R}_{2}^{-1}
\end{array}\right]
$$

where $\mathbf{0}$ is the $2 \times 2$ zero matrix and $\mathbf{R}_{2}$ is defined as the $2 \times 2$ right-lower block of the measurement noise covariance matrix, that is $\mathbf{R}_{2} \triangleq \operatorname{diag}\left(\sigma_{V}^{2}, \sigma_{\phi}^{2}\right)$.

In Eq. (42), there are two main issues: first, one has to show that the expectations in the submatrix $\Pi$ exist, and then one has to compute them. These expectations are taken over non-linear functions of the state random variables, which makes it hard to find a closed-form expression of the PCRLB. This is a well known issue, as pointed out in [42], where after arguing the difficulty of deriving a PCRLB in cartesian coordinates, as we do, the PCRLB has been derived by using logarithmic polar coordinates. However, we note that the model adopted in [42] does not not include speed and orientation measurements by on-board sensors, and that the derivation of the PCRLB in a coordinate system does not give insight on which is the best estimator on another coordinate system [37], [41], which greatly limits the model of [42] for our case. In the cartesian coordinate system one could resolve the issue by a Monte Carlo approach to calculate numerically the PCRLB. However, analytical expressions of the PCRLB are of fundamental interest for many localization applications, such as when trying to understand where to place the slaves in order to maximize the information content given by the Fisher information matrix [43], [44], or when planning the path of a robot to minimize the uncertainty in its location. This further motivates the derivation of the analytical results that we present below.

We must first prove that the expectations in the submatrix $\Pi$ exist. In the following, we establish the existence of such expectations by deriving them in closed-form, and we derive closed-form expressions of upper and lower bounds for the entries of $\Pi$ for which the exact closed form expression is too complex for practical numerical evaluations. Then, we calculate them numerically in Section V. To compute the expectations on the diagonal elements of $\Pi$, we establish the following result.

Proposition 4.2: Let $q$ and $z$ be independent Gaussian random variables with average and standard deviation $\mu_{q}, \sigma_{q}$ and $\mu_{z}$ and $\sigma_{z}$, respectively, with $\sigma_{z}=\sigma_{q}{ }^{1}$. Then,

$$
\mathbb{E}\left\{\frac{q^{2}}{q^{2}+z^{2}}\right\}=\frac{1}{1+\Upsilon}\left[1+\sum_{k=1}^{\infty}(-1)^{k} \frac{\mu_{F, k}}{(1+\Upsilon)^{k}}\right],
$$

where

$$
\Upsilon \triangleq \frac{\mu_{z}^{2}+\sigma_{z}^{2}}{\mu_{q}^{2}+\sigma_{q}^{2}}\left[1+\sum_{k=1}^{\infty}(-1)^{k} \frac{\sigma_{q}^{2 k} \mu_{k}}{\left(\mu_{q}^{2}+\sigma_{q}^{2}\right)^{k}}\right],
$$

and $\mu_{k}$ denotes the $k$-th central moment of the noncentral chi-squared distribution of 1 degree of freedom, and $\mu_{F, k}$

\footnotetext{
${ }^{1}$ Recall that these are the variances of the process noise on the coordinates, see Eq.(29).
} 


$$
\begin{aligned}
& \mathbf{D}_{k}^{11}=\mathbb{E}\left\{\left[\begin{array}{cccc}
\sigma_{1}^{-2} & 0 & \frac{T}{\sigma_{1}^{2}} \cos \phi_{k} & -\frac{T}{\sigma_{1}^{2}} V_{k} \sin \phi_{k} \\
0 & \sigma_{2}^{-2} & \frac{T}{\sigma_{2}^{2}} \sin \phi_{k} & \frac{T}{\sigma_{2}^{2}} V_{k} \cos \phi_{k} \\
\frac{T}{\sigma_{1}^{2}} \cos \phi_{k} & \frac{T}{\sigma_{2}^{2}} \sin \phi_{k} & \frac{T^{2}}{\sigma_{1}^{2}} \cos ^{2} \phi_{k}+\frac{T^{2}}{\sigma_{2}^{2}} \sin ^{2} \phi_{k}+\sigma_{3}^{-2} & T^{2}\left(\frac{\sigma_{1}^{2}-\sigma_{2}^{2}}{\sigma_{1}^{2} \sigma_{2}^{2}}\right) V_{k} \cos \phi_{k} \sin \phi_{k} \\
-\frac{T}{\sigma_{1}^{2}} V_{k} \sin \phi_{k} & \frac{T}{\sigma_{2}^{2}} V_{k} \cos \phi_{k} & T^{2}\left(\frac{\sigma_{1}^{2}-\sigma_{2}^{2}}{\sigma_{1}^{2} \sigma_{2}^{2}}\right) V_{k} \cos \phi_{k} \sin \phi_{k} & \frac{T^{2}}{\sigma_{1}^{2}} V_{k}^{2} \sin ^{2} \phi_{k}+\frac{T^{2}}{\sigma_{2}^{2}} V_{k}^{2} \cos ^{2} \phi_{k}+\sigma_{4}^{-2}
\end{array}\right]\right\} \\
& =\left[\begin{array}{cccc}
\sigma_{1}^{-2} & 0 & \frac{T c_{0}}{\sigma_{1}^{2}} \varepsilon_{k} & -\frac{T V_{0} s_{0}}{\sigma_{1}^{2}} \varepsilon_{k} \\
0 & \sigma_{2}^{-2} & \frac{T s_{0}}{\sigma_{2}^{2}} \varepsilon_{k} & \frac{T V_{0} c_{0}}{\sigma_{2}^{2}} \varepsilon_{k} \\
\frac{T c_{0}}{\sigma_{1}^{2}} \varepsilon_{k} & \frac{T s_{0}}{\sigma_{2}^{2}} \varepsilon_{k} & T^{2}\left(\frac{\sigma_{1}^{2}+\sigma_{2}^{2}}{2 \sigma_{1}^{2} \sigma_{2}^{2}}+\frac{\sigma_{2}^{2}-\sigma_{1}^{2}}{2 \sigma_{1}^{2} \sigma_{2}^{2}} c_{0}^{\prime} \varepsilon_{k}^{4}\right)+\sigma_{3}^{-2} & T^{2}\left(\frac{\sigma_{1}^{2}-\sigma_{2}^{2}}{\sigma_{1}^{2} \sigma_{2}^{2}}\right) \frac{V_{0} s_{0}^{\prime}}{2} \varepsilon_{k}^{4} \\
-\frac{T V_{0} s_{0}}{\sigma_{1}^{2}} \varepsilon_{k} & \frac{T V_{0} c_{0}}{\sigma_{2}^{2}} \varepsilon_{k} & T^{2}\left(\frac{\sigma_{1}^{2}-\sigma_{2}^{2}}{\sigma_{1}^{2} \sigma_{2}^{2}}\right)^{\frac{V_{0} s_{0}^{\prime}}{2}} \varepsilon_{k}^{4} & T^{2}(k-1) \sigma_{3}^{2}\left(\frac{\sigma_{1}^{2}+\sigma_{2}^{2}}{2 \sigma_{1}^{2} \sigma_{2}^{2}}+\frac{\sigma_{1}^{2}-\sigma_{2}^{2}}{2 \sigma_{1}^{2} \sigma_{2}^{2}} c_{0}^{\prime} \varepsilon_{k}^{4}\right)+\sigma_{4}^{-2}
\end{array}\right] \\
& \boldsymbol{\Pi}=\mathbb{E}\left\{\left[\begin{array}{cc}
\sigma_{r_{1}}^{-2} d_{1,1}^{2}+\ldots+\sigma_{r_{M}}^{-2} d_{1, M}^{2} & \sigma_{r_{1}}^{-2} d_{1,1} d_{2,1}+\ldots+\sigma_{r_{M}}^{-2} d_{1, M} d_{2, M} \\
\sigma_{r_{1}}^{-2} d_{1,1} d_{2,1}+\ldots+\sigma_{r_{M}}^{-2} d_{1, M} d_{2, M} & \sigma_{r_{1}}^{-2} d_{2,1}^{2}+\ldots+\sigma_{r_{M}}^{-2} d_{2, M}^{2}
\end{array}\right]\right\} .
\end{aligned}
$$

denotes the $k$-th central moment of the doubly noncentral Fdistribution.

Proof: See Appendix G.

The previous proposition allows us to establish the existence of the expectations on the diagonal elements of $\Pi$. A similar result can be derived for the off-diagonal elements. Unfortunately, these closed form expressions are of limited practical usage due to the computational complexity of the many summations and function evaluations therein involved. Accordingly, we derive the following upper and lower bounds, which are much more easy to use in practice:

Proposition 4.3: Let $q$ and $z$ be independent Gaussian random variables of average and standard deviation $\mu_{q}, \sigma_{q}$ and $\mu_{z}$ and $\sigma_{z}$, respectively. Then

$$
e^{\alpha-\ln \left(\sigma_{q}^{2}+\mu_{q}^{2}+\sigma_{z}^{2}+\sigma_{z}^{2}\right)} \leq \mathbb{E}\left\{\frac{q^{2}}{q^{2}+z^{2}}\right\} \leq 1,
$$

where $\alpha=-\gamma_{e}-\ln (2)-2 \ln \left(\sigma_{q}\right)-M\left(0,1 / 2,-\mu_{q}^{2} / 2 \sigma_{q}^{2}\right)$, the symbol $\gamma_{e}$ denotes the Euler Gamma constant, and the symbol $M(a, b, z)$ denotes Kummer's confluent hypergeometric function [45].

Proof: See Appendix H.

To compute bounds on the off-diagonal elements of $\Pi$, we give the following proposition:

Proposition 4.4: Let $q$ and $z$ be independent Gaussian random variables with average and standard deviation $\mu_{q}, \sigma_{q}$, and $\mu_{z}$ and $\sigma_{z}$, respectively. Let the expectation be computed in the Cauchy principal value sense. Then

$$
-\frac{1}{2} \leq \mathbb{E}\left\{\frac{q z}{q^{2}+z^{2}}\right\} \leq \frac{1}{2},
$$

Proof: The bounds follow immediately from that $-1 / 2 \leq$ $q z /\left(q^{2}+z^{2}\right) \leq 1 / 2, \forall q \in \mathbb{R}, \forall z \in \mathbb{R}$.

We are now in the position to compute the upper and lower bounds on the Fisher information matrix, in the positive semidefinite sense, by the following result.

Proposition 4.5: Let $\mathbf{J}^{(u b)}$ and $\mathbf{J}^{(l b)}$ be two matrices consisting of element-wise upper and lower bounds, respectively, of the Fisher information matrix $\mathbf{J}$ in Eq. (31) as obtained by
Propositions 4.3 and 4.4 for the elements of $\Pi$ of Eq. (42). Let

$$
\begin{aligned}
& \mathbf{J}^{(u b, G) \triangleq} \begin{cases}\mathbf{J}_{i i}^{(u b)}, & \text { if } \quad \mathbf{J}_{i i}^{(u b)}>\min \left(u_{i}^{(\text {row })}, u_{i}^{(c o l)}\right) \\
\min \left(u_{i}^{(\text {row })},\right. & \left.\left.u_{i}^{(c o l)}\right)+\epsilon,{ }^{2}\right)\end{cases} \\
& \mathbf{J}_{i i}^{(u b, G)} \triangleq\left\{\min \left(u_{i}^{(\text {row })}, u_{i}^{(c o l)}\right)+\epsilon,\right. \\
& \text { if } \mathbf{J}_{i i}^{(u b)} \leq \min \left(u_{i}^{(\text {row })}, u_{i}^{(c o l)}\right) \\
& \mathbf{J}_{i j}^{(u b, G)} \triangleq \mathbf{J}_{i j}^{(u b)}, \quad \forall i, j=1 \ldots n, \text { with } i \neq j, \\
& \mathbf{J}_{i i}^{(l b, G)} \triangleq \mathbf{J}_{i i}^{(l b)}, \quad \forall i=1 \ldots n, \\
& \mathbf{J}_{i j}^{(l b, G)} \triangleq\left\{\begin{array}{l}
\mathbf{J}_{i j}^{(u b)} \quad \text { if } \mathbf{J}_{i i}^{(l b)}>\min \left(l_{i}^{(\text {row })}, l_{i}^{(c o l)}\right) \\
\left(\min \left(\mathbf{J}_{i i}^{(l b)} / l_{i}^{(\text {row })}, \mathbf{J}_{i i}^{(l b)} / l_{i}^{(c o l)}\right)-\epsilon\right) \mathbf{J}_{i j}^{(l b)} \\
\text { if } \mathbf{J}_{i i}^{(l b)} \leq \min \left(l_{i}^{(\text {row })}, l_{i}^{(c o l)}\right)
\end{array}\right. \\
& \forall i, j=1 \ldots n, \text { with } i \neq j \text {, }
\end{aligned}
$$

where $u_{i}^{(\text {row })}=\sum_{j \neq i}\left|\mathbf{J}_{i j}^{(u b)}\right|, u_{i}^{(\text {col })}=\sum_{j \neq i}\left|\mathbf{J}_{j i}^{(u b)}\right|, l_{i}^{(\text {row })}=$ $\sum_{j \neq i}\left|\mathbf{J}_{i j}^{(l b)}\right|, l_{i}^{(c o l)}=\sum_{j \neq i}\left|\mathbf{J}_{j i}^{(l b)}\right|$ and $\epsilon$ is an arbitrary constant with $\epsilon>0$. Then

$$
\mathbf{0} \preceq \mathbf{J}^{(l b, G)} \preceq \mathbf{J} \preceq \mathbf{J}^{(u b, G)} .
$$

Proof: See Appendix I.

This concludes the steps to establish the existence and upper and lower bounds of the Fisher information matrix. In the following section, we use these results to compare the performance of our proposed localization method to the CRLB.

\section{Simulation Results}

In this section we present simulation results to validate both the PCRLB derivations and the estimator we presented in the previous sections. In particular, we compare our new estimator based on Pareto optimization that we have developed in Section III to some solutions from the literature and to the PCRLB we derived in Section IV. 


\section{A. Performance of the proposed sensor fusion method}

In this section we present extensive simulation results of our new estimator (8) based on the optimal fusion coefficient of Eq. (25) and Pareto weighting factor of Eq. (27). The values of the parameters used in the numerical simulations are the following:

- Speed measurement noise standard deviation $\sigma_{v}=$ $0.05 \mathrm{~m} / \mathrm{s}$, to model the worst-case performance of an odometry sensor, [35];

- Orientation measurement noise standard deviation $\sigma_{\phi}=$ $\pi / 8 \mathrm{rad}$, to model the worst-case performance of a magnetometer subject to disturbances due to the environment, see, e.g., [36], [46];

- Ranging noise model parameters (in Eq. (2)): $\sigma_{0}=0.25$, $\kappa_{\sigma}=0.25$

- Sampling time interval $T=0.1 \mathrm{~s}$.

The following two scenarios have been considered:

(A) Linear trajectory with constant speed of $0.1 \mathrm{~m} / \mathrm{s}$. This is a deterministic trajectory, that is, no process noise is added. As an example, this scenario emulates the real-world situation in which the mobile node is on a vehicle moving along a straight line without performing any maneuver.

(B) Piece-wise-linear (PWL)-acceleration trajectory, which is more complex and realistic than the linear one, with a maximum acceleration of $0.5 \mathrm{~m} / \mathrm{s}^{2}$, see Fig. 5. This scenario emulates a mobile node performing maneuvers with an acceleration range consistent with the indoor environment, e.g. a walking person carrying the mobile node.

The absolute value of $\beta^{*}$, provided by the algorithm, has been clipped to 0.99 to ensure that the average of the estimation error is non-expansive, as discussed in Section III.

Note that, in 3.7, in the expression for $\gamma$ the true speed $V_{k}$ and orientation $\phi_{k}$ terms are present. Since these are not available, we use these approximations:

$$
\begin{aligned}
V_{\text {approx }}(k) \simeq & \frac{1}{T}\left(\left(\hat{x}_{1, k \mid k}-\hat{x}_{1, k-1 \mid k-1}\right)^{2}\right. \\
& \left.+\left(\hat{x}_{2, k \mid k}-\hat{x}_{2, k-1 \mid k-1}\right)^{2}\right)^{\frac{1}{2}}, \\
\phi_{\text {approx }}(k) \simeq & \arctan \left(\frac{\hat{x}_{2, k \mid k}-\hat{x}_{2, k-1 \mid k-1}}{\hat{x}_{1, k \mid k}-\hat{x}_{1, k-1 \mid k-1}}\right) .
\end{aligned}
$$

Also, note that the second-order moment of the ranging error, given by (17), depends on the true ranges $r_{i}$. Therefore, for the calculation of the term $\mathbb{E}\left\{\left(w_{x_{1, k+1}}^{(r)}\right)^{2}\right\}$ in $b_{k}$ and $a_{k}$, we use the approximations for the ranges

$$
r_{i, k+1}^{\text {(approx) }}=\sqrt{\left(s_{i, 1}-\hat{x}_{k}\right)^{2}+\left(s_{i, 2}-\hat{y}_{k}\right)^{2}}, \quad i=1, \ldots, M .
$$

The absolute error and empirical Cumulative Distribution Function (CDF) obtained by our new method for scenario A are shown in Fig. 3 and 4. In this scenario, a root-meansquared error (RMSE) of $4.0 \mathrm{~cm}$ in the position estimate over the entire trajectory has been obtained by our method. Furthermore, in Fig. 5 the more realistic PWL-acceleration case is shown, where an RMSE of $5.5 \mathrm{~cm}$ is obtained.
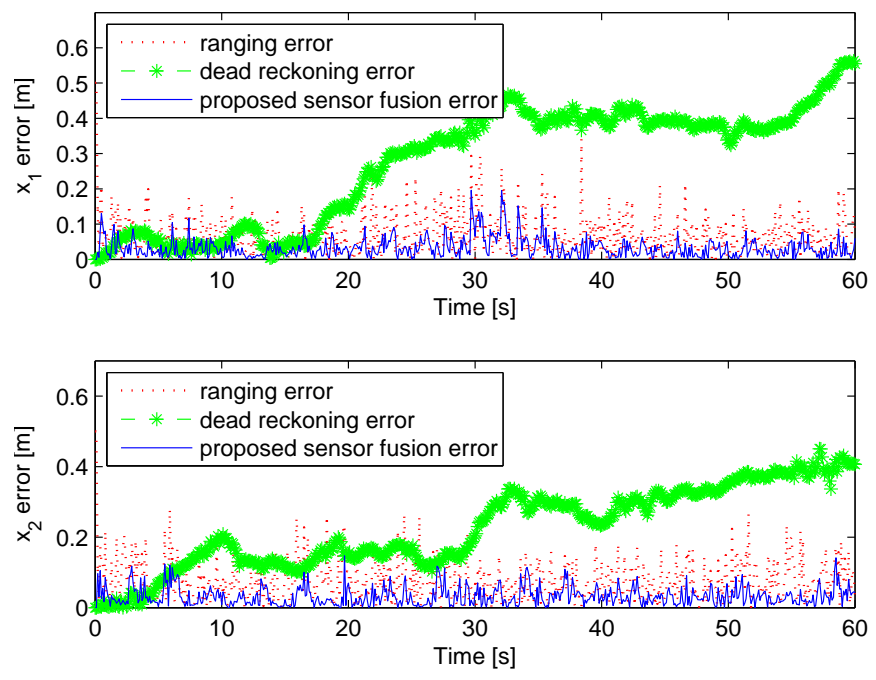

Fig. 3. Absolute errors in scenario A. The dead-reckoning estimates, asterisks, have a high bias, while the WLS ranging estimates, dashed line, present a higher variance, but a reduced bias. The proposed sensor fusion technique is able to reach a good tradeoff between estimator bias and variance.

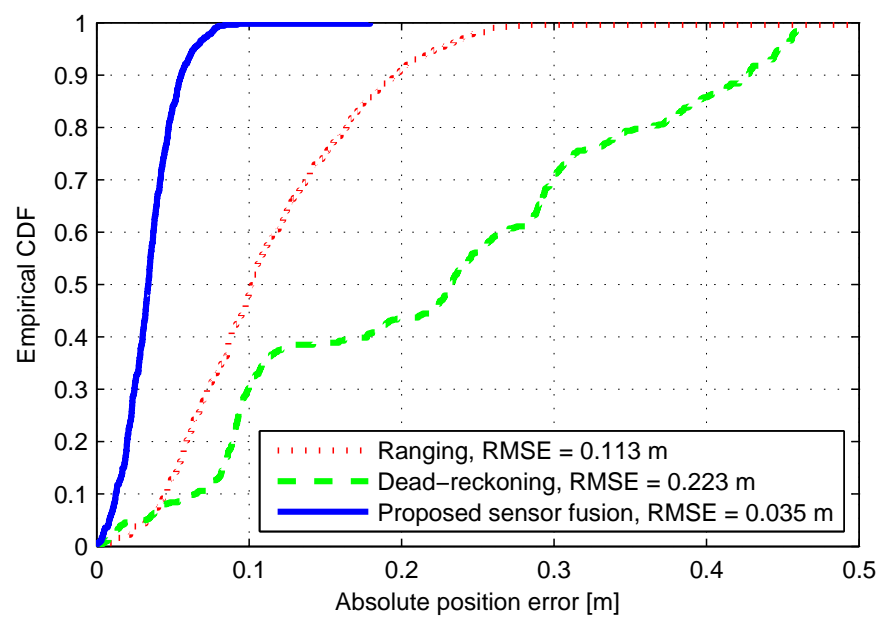

Fig. 4. Empirical CDF of the absolute positioning error in $2 \mathrm{D}$ for scenario $\mathrm{A}$ Using the proposed method (blue line), about $95 \%$ of the position estimates have an error smaller than $7 \mathrm{~cm}$, and the performance is improved with respect to both the ranging and the dead-reckoning stand-alone systems.

Numerical simulation results show that the approximations (49)-(51) give good performance and that the proposed method yields a good trade-off between variance and bias of the estimated position. Therefore, the proposed estimator overcomes the limitation of dead reckoning (that is the slowly accumulating bias) while also reducing the relatively higher variance of the ranging estimator. The result is an overall smooth and accurate estimate of the trajectory. In the following, we compare the proposed estimator to other solutions that are commonly adopted in similar localization problems.

\section{B. Performance Comparison}

Here we compare the sensor fusion based on Pareto optimization proposed in this paper to several other methods in the two mobility scenarios considered in the previous section. To provide an extensive comparison, the simulations 


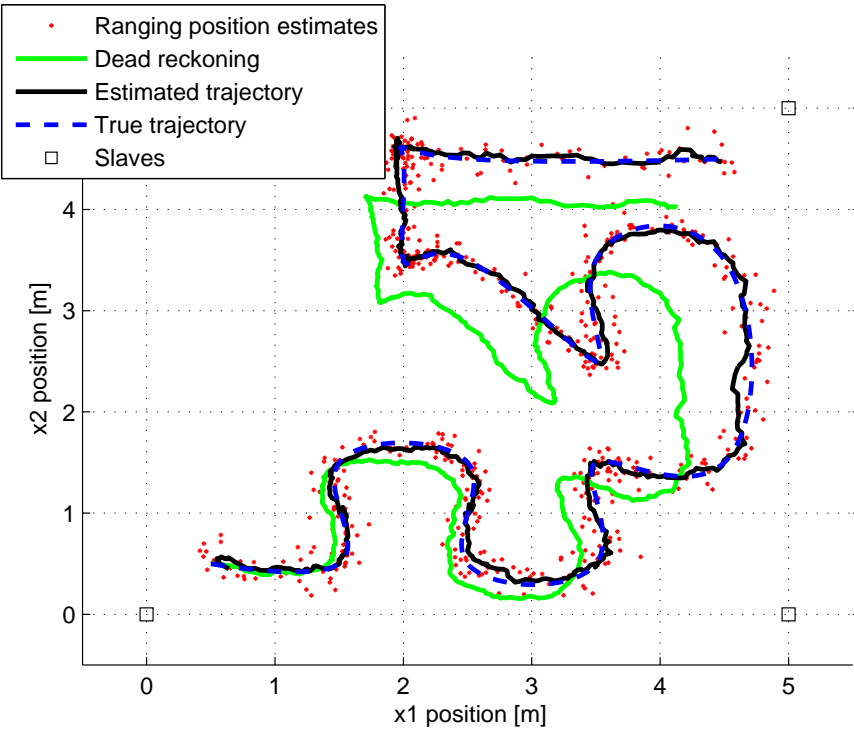

Fig. 5. PWL-acceleration trajectory case, scenario B. The proposed sensor fusion technique closely tracks the true trajectory with $\mathrm{RMSE}=5.5 \mathrm{~cm}$.

TABLE I

Average Execution Time Per Iteration [s].

Matlab ${ }^{\circledR}$ implementation on an Intel ${ }^{\circledR}$ Core2 Duo, $2.40 \mathrm{GHz}$ PC with $3 \mathrm{~GB}$ RAM.

\begin{tabular}{cc}
\hline$E K F$ & $1.3 \times 10^{-4}$ \\
\hline$M S E$ Eq. (26) & $1.7 \times 10^{-4}$ \\
\hline$L C$ - $K F$ & $1.9 \times 10^{-4}$ \\
\hline$U K F$ & $4.2 \times 10^{-4}$ \\
\hline Proposed sensor fusion, Eq. (25) & $4.5 \times 10^{-4}$ \\
\hline \hline
\end{tabular}

are performed for different values of the sampling period $T$ and of the speed $V$ for scenario A, whereas for scenario B the simulations are performed for different values of $T$ and of the maximum acceleration. Moreover, for each configuration, the simulations are performed for 10 realizations of the random noises and the resulting RMSE values are averaged.

The sensor fusion methods available from the literature that we have considered are the following:

- Extended Kalman Filter (EKF) [25], where the measured speed and orientation data are employed as inputs, and the ranges as measurements. No motion model is assumed.

- Unscented Kalman Filter (UKF) [47], with the same state-space model as the EKF above.

- Loosely Coupled Kalman Filter (LC-KF), where range measurements are preprocessed with the WLS algorithm to obtain preliminary position measurements, thus using a linear measurement equation. The usual linearization of the state function is performed for calculating the input noise matrix [25].

Notice that the methods based on the Kalman filter theory have been implemented without assumptions about the motion model, to provide a fair comparison with the developed method of this paper. If prior information about motion models is included in the state space formulation, the Kalman approaches might be able to provide better performance, as we will show in the example in the next section. Notice also that we provide results of the MSE special case when $\rho_{1, k}=0.5$ $\forall k$ in Proposition 3.7. This method does not provide good

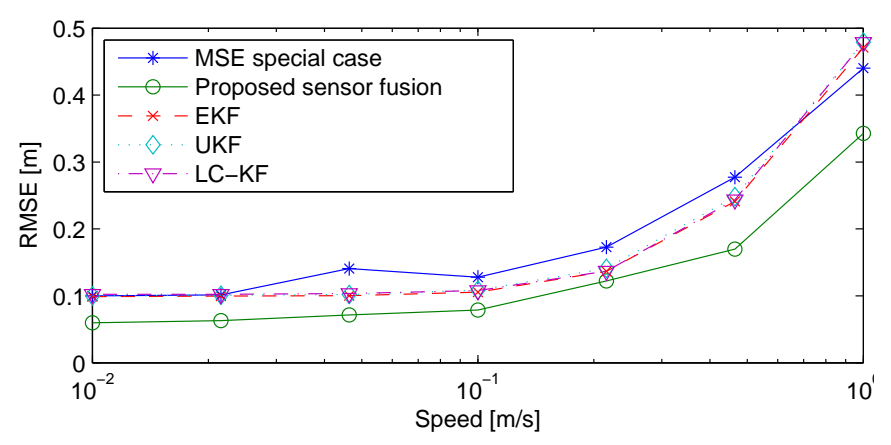

Fig. 6. RMSE vs speed of the different methods for Scenario A with $\mathrm{T}=0.5 \mathrm{~s}$.

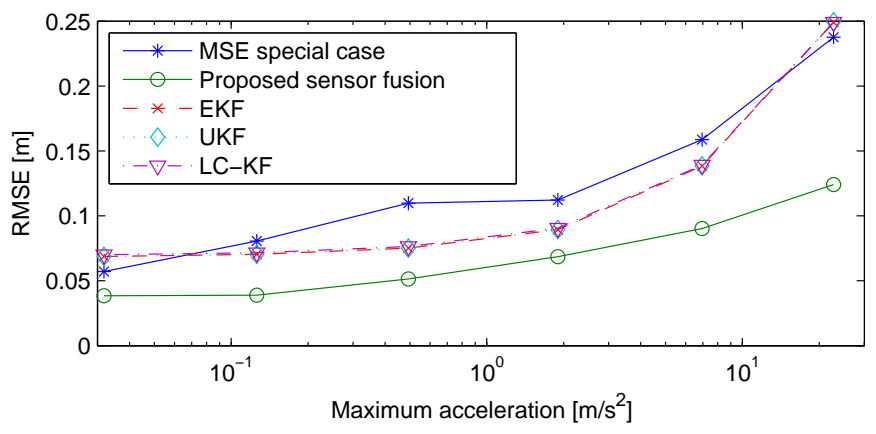

Fig. 7. RMSE vs maximum acceleration of several methods for Scenario B with $\mathrm{T}=0.1 \mathrm{~s}$.

performance compared to the more general case of choosing the best $\rho_{1, k}$ by problem (27). However, it features a much smaller computational complexity.

Comparisons for two selected simulation configurations are provided in Fig. 6 and 7, as obtained in the two scenarios with different values of $T$. The proposed sensor fusion based on Pareto optimization outperforms the other methods, while the KF-based methods have similar performance among them. However, the proposed sensor fusion based on Pareto optimization requires the highest computational load among the implemented techniques, as shown in the average simulation execution times listed in Table I, although it is close to the execution time of UKF.

\section{CRLB evaluation}

In this section, we provide a comparison of the RMSE performance of the proposed sensor fusion method based on Pareto optimization and the Kalman filter based methods we used in the previous section to the fundamental limits given by the Cramér Rao lower bound. In particular, we first provide comparisons with the ParCRLB, and then with the PCRLB as derived in Section IV.

The performance of the filters was compared based on the RMSE of the position estimate, where the error is defined as the Euclidean distance between the true position and the estimate. The results compared to the ParCRLB for several specific trajectories are presented in Fig. 8, where the error curves were obtained by averaging over 100 Monte-Carlo runs. We notice that the proposed sensor fusion method based on Pareto optimization provides better performance than the other considered KF methods as it is the closest to the ParCRLB. We 

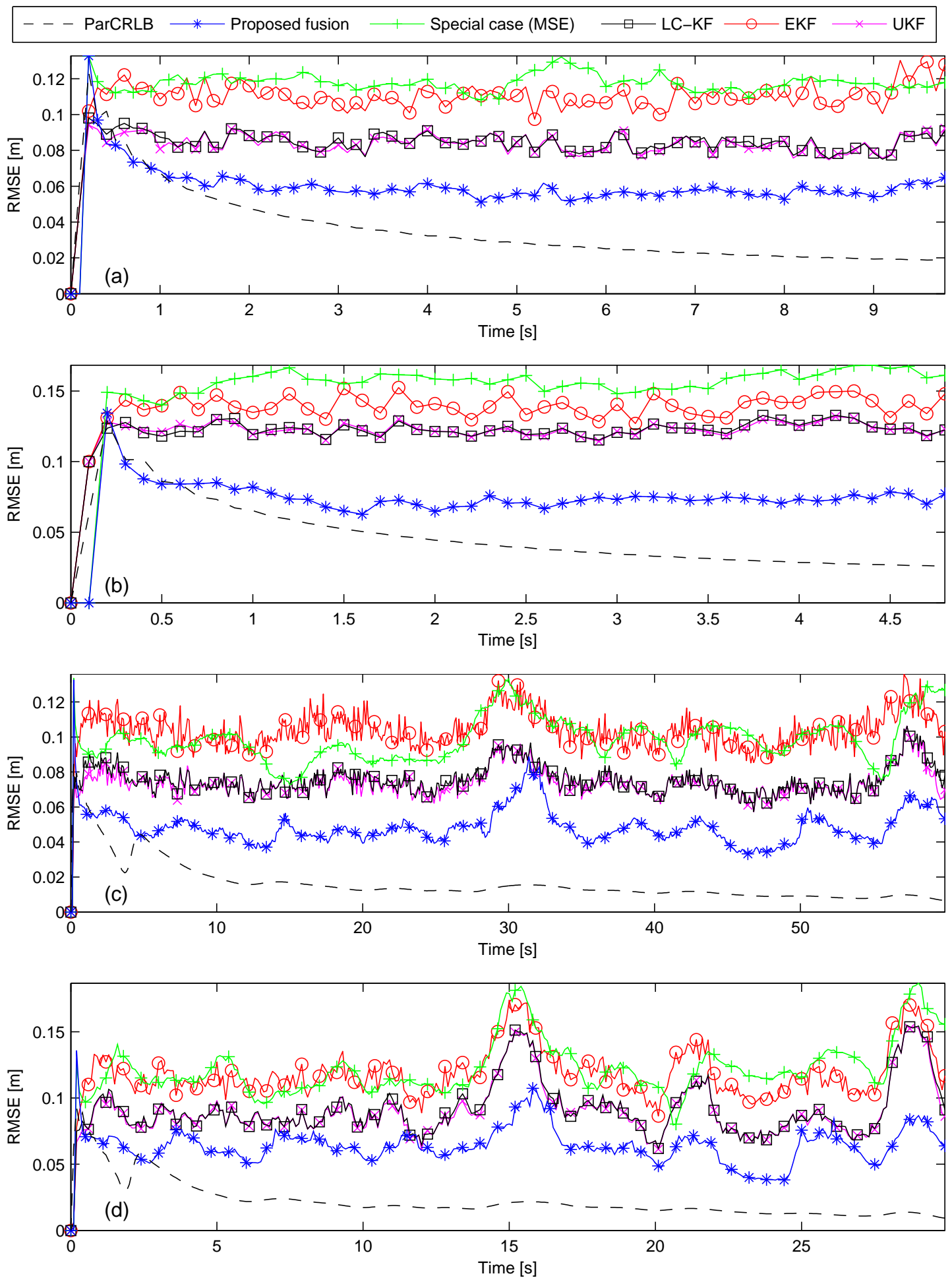

Fig. 8. Error performance of the proposed sensor fusion method based on Pareto optimization of Eq. (25) and other methods against the theoretical bound given by the square root of the trace of the ParCRLB (dashed line): (a) linear trajectory, constant velocity of 0.5 [m/s]; (b) linear trajectory, constant velocity of $1[\mathrm{~m} / \mathrm{s}]$; (c) spline trajectory, maximum acceleration of $0.3\left[\mathrm{~m} / \mathrm{s}^{2}\right]$; (d) spline, maximum acceleration of $1\left[\mathrm{~m} / \mathrm{s}^{2}\right]$. 


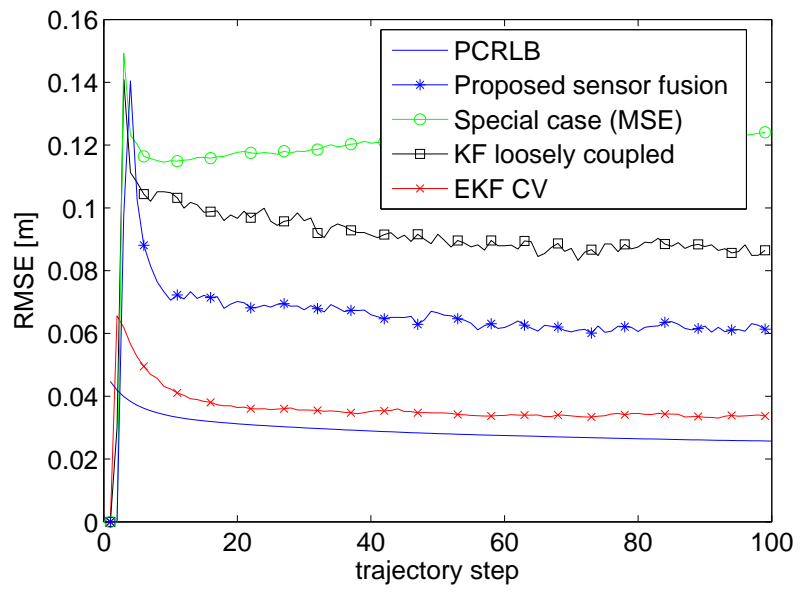

Fig. 9. Numerical simulation results on the class of trajectories defined by the model in (29)-(30). The behavior of the proposed sensor fusion method based on Pareto optimization is compared to other estimators from the literature and to the PCRLB derived in Section IV. Error curves are obtained as the average of 1000 Monte Carlo iterations.

stress that none of the considered methods makes assumptions about the motion model, therefore in this sense the ParCRLB is an overly optimistic bound.

Furthermore, the numerical simulation results related to the model in (29)-(30) are shown in Fig. 9, in comparison with the derived PCRLB in (31). The method denoted as "EKF CV" is implemented using the motion model in (29), and the same model is used to generate the trajectories. Specifically, a standard EKF is implemented [25], by linearizing about the current estimate of the mean and covariance, where the Jacobian matrices are computed by numerical differentiation at each iteration. In this case, we notice that EKF CV outperforms the proposed sensor fusion method based on Pareto optimization and approaches the PCRLB. This confirms that the knowledge of the specific underlying motion model is beneficial for estimation. However, the proposed sensor fusion method still provides good performance, improving over the LC-KF. Conversely to EKF CV, the proposed method does not assume any specific motion model, thus it can be applied to a wider class of trajectories, proving to be robust with respect to model mismatch. Nevertheless, the same Pareto optimization approach presented in this paper could be applied to derive an estimator which exploits the knowledge of the motion model, although such derivation is outside of the scope of this paper.

\section{CONCLUSION}

In this paper, the fundamental limitations of position estimation for a mobile node by the fusion of information from ranging, velocity and angular measurements were investigated. Upper and lower bounds of the posterior Cramér-Rao lower bound were derived. A sensor fusion method based on Pareto optimization was proposed. The analysis was validated by Monte Carlo simulations, which showed that the proposed method provides better performance than Kalman filteringbased methods when no knowledge of the dynamic model of the underlying system is assumed or when the model is time varying.
Future work includes the extension of the proposed estimator to cases when the mobility model is known. The good performance achieved by the proposed sensor fusion method based on Pareto optimization in the case of no motion model assumption lets us think that the use of a motion model would substantially farther improve the performance of the proposed method.

\section{APPENDIX A}

PROOF OF LEMMA 3.2

By taking the mean of Eq. (16), we obtain:

$$
\begin{aligned}
\mathbb{E}\left\{\hat{\mathbf{x}}^{(r)}\right\}= & \left(\mathbf{A}^{T} \mathbf{W A}\right)^{-1} \mathbf{A}^{T} \mathbf{W} \\
& \times\left(\mathbf{1} \mathbb{E}\left\{\tilde{r}_{M}^{2}\right\}-\mathbb{E}\left\{\left[\tilde{r}_{1}^{2}, \ldots, \tilde{r}_{M-1}^{2}\right]^{T}\right\}+\mathbf{a}\right) \\
= & \mathbf{x}+\left(\mathbf{A}^{T} \mathbf{W A}\right)^{-1} \mathbf{A}^{T} \mathbf{W} \\
& \times\left(\mathbf{1} \sigma_{w_{r_{M}}}^{2}-\left[\sigma_{w_{r_{1}}}^{2}, \ldots, \sigma_{w_{r_{M-1}}}^{2}\right]^{T}\right) \\
= & \mathbf{x}+\mathbb{E}\left\{\mathbf{w}_{r}\right\}
\end{aligned}
$$

whereby the lemma follows.

\section{APPENDIX B}

PROOF OF LEMMA 3.3

By taking the square of Eq. (1) we get $\tilde{r}_{i}^{2}=r_{i}^{2}+w_{r_{i}}^{2}+$ $2 r_{i} w_{r_{i}}$. Then, using this expression in (14) and substituting in (16), we have the following expression for the ranging-based estimate:

$$
\begin{aligned}
\hat{\mathbf{x}}^{(r)}= & \left(\mathbf{A}^{T} \mathbf{W A}\right)^{-1} \mathbf{A}^{T} \mathbf{W}\left\{\left[r_{M}^{2}-r_{1}^{2}, \ldots, r_{M}^{2}-r_{M-1}^{2}\right]^{T}\right. \\
& \left.+\mathbf{B}_{k}+\mathbf{a}\right\},
\end{aligned}
$$

where

$$
\mathbf{B}_{k}=\left[\begin{array}{c}
w_{r_{M}}^{2}+2 r_{M} w_{r_{M}}-w_{r_{1}}^{2}-2 r_{1} w_{r_{1}} \\
\vdots \\
w_{r_{M}}^{2}+2 r_{M} w_{r_{M}}-w_{r_{M-1}}^{2}-2 r_{M-1} w_{r_{M-1}}
\end{array}\right] .
$$

Therefore the error can be expressed as $\mathbf{w}_{r}=$ $\left(\mathbf{A}^{T} \mathbf{W A}\right)^{-1} \mathbf{A}^{T} \mathbf{W} \mathbf{B}_{k}$, and its correlation is $\mathbb{E}\left\{\mathbf{w}_{r}^{2}\right\}=$ $\left(\mathbf{A}^{T} \mathbf{W A}\right)^{-1} \mathbf{A}^{T} \mathbf{W} \mathbb{E}\left\{\mathbf{B}_{k} \mathbf{B}_{k}^{T}\right\} \mathbf{W}^{T} \mathbf{A}\left(\mathbf{A}^{T} \mathbf{W A}\right)^{-1}$. We let $\mathbf{C} \triangleq \mathbb{E}\left\{\mathbf{B}_{k} \mathbf{B}_{k}^{T}\right\}$, whereas the lemma follows after some algebraic manipulation.

\section{APPENDIX C \\ PROOF OF LEMMA 3.4}

Since $\tilde{V}_{k}$ and $\tilde{\phi}_{k}$ are independent,

$$
\begin{aligned}
\mathbb{E}\left\{\tilde{V}_{k} \cos \left(\tilde{\phi}_{k}\right)\right\}= & \mathbb{E}\left\{\tilde{V}_{k}\right\} \mathbb{E}\left\{\cos \left(\tilde{\phi}_{k}\right)\right\} \\
= & V_{k} \mathbb{E}\left\{\cos \left(\phi_{k}+w_{\phi_{k}}\right)\right\} \\
= & V_{k}\left(\cos \left(\phi_{k}\right) \mathbb{E}\left\{\cos \left(w_{\phi, k}\right)\right\}\right. \\
& \left.-\sin \left(\phi_{k}\right) \mathbb{E}\left\{\sin \left(w_{\phi, k}\right)\right\}\right) .
\end{aligned}
$$


By using the cosine series,

$\mathbb{E}\left\{\cos \left(w_{\phi, k}\right)\right\}=\mathbb{E}\left\{1-\frac{w_{\phi, k}^{2}}{2}+\frac{w_{\phi, k}^{4}}{4 !}+\cdots+(-1)^{n} \frac{w_{\phi, k}^{2 n}}{(2 n) !}\right\}$ $=1-\frac{\sigma_{\phi}^{2}}{2}+\frac{\sigma_{\phi}^{4}}{2^{2} \cdot 2 !}+\cdots+(-1)^{n} \frac{\sigma_{\phi}^{2 n}}{2^{n} \cdot n !}=e^{-\frac{\sigma_{\phi}^{2}}{2}}$

where we have used that $\tilde{\phi}_{k}$ is Gaussian. Similarly, by using the sine series, it results

$$
\begin{aligned}
\mathbb{E}\left\{\sin \left(w_{\phi, k}\right)\right\}= & \mathbb{E}\left\{w_{\phi}-\frac{w_{\phi, k}^{3}}{3 !}+\frac{w_{\phi, k}^{5}}{5 !}-\frac{w_{\phi, k}^{7}}{7 !}\right. \\
& \left.+\cdots+(-1)^{n} \frac{w_{\phi_{k}}^{2 n+1}}{(2 n+1) !}\right\}=0
\end{aligned}
$$

Then Eq. (18) follows by substituting Eqs. (53) and (54) in Eq. (52). Furthermore, since $\tilde{V}_{k}$ and $\tilde{\phi}_{k}$ are independent, we have

$$
\mathbb{E}\left\{\tilde{V}_{k}^{2} \cos ^{2}\left(\tilde{\phi}_{k}\right)\right\}=\mathbb{E}\left\{\tilde{V}_{k}^{2}\right\} \mathbb{E}\left\{\cos ^{2}\left(\tilde{\phi}_{k}\right)\right\}
$$

For the second factor of the right-end side of this expression we have

$$
\begin{aligned}
\mathbb{E}\left\{\cos ^{2}\left(\tilde{\phi}_{k}\right)\right\}= & \mathbb{E}\left\{\frac{1}{2}+\frac{1}{2} \cos \left(2 \tilde{\phi}_{k}\right)\right\} \\
= & \frac{1}{2}+\frac{1}{2} \mathbb{E}\left\{\cos \left(2\left(\phi_{k}+w_{\phi, k}\right)\right)\right\} \\
= & \frac{1}{2}+\frac{1}{2} \mathbb{E}\left\{\cos \left(2 \phi_{k}\right) \cos \left(2 w_{\phi, k}\right)\right. \\
& \left.-\sin \left(2 \phi_{k}\right) \sin \left(2 w_{\phi, k}\right)\right\} \\
= & \frac{1}{2}+\frac{1}{2} \cos \left(2 \phi_{k}\right) \mathbb{E}\left\{\cos \left(2 w_{\phi, k}\right)\right\}
\end{aligned}
$$

Using the cosine series, we write

$$
\begin{aligned}
\mathbb{E}\left\{\cos \left(2 w_{\phi, k}\right)\right\}= & \mathbb{E}\left\{1-2^{2} \frac{w_{\phi_{k}}^{2}}{2 !}+2^{4} \frac{3 w_{\phi_{k}}^{4}}{4 !}\right. \\
& \left.-2^{6} \frac{5 w_{\phi_{k}}^{6}}{6 !}+\cdots+(-1)^{n} \frac{\left(2 w_{\phi}\right)^{2 n}}{(2 n) !}\right\} \\
= & 1-2 \frac{\sigma_{\phi}^{2}}{1 !}+2^{2} \frac{\sigma_{\phi}^{4}}{2 !}-2^{3} \frac{\sigma_{\phi}^{6}}{3 !} \\
& +\cdots+(-1)^{n} \frac{2^{n} \sigma_{\phi}^{2 n}}{n !}=e^{-2 \sigma_{\phi}^{2}} .
\end{aligned}
$$

By substituting (57) in (56), we obtain:

$$
\mathbb{E}\left\{\cos ^{2}\left(\tilde{\phi}_{k}\right)\right\}=\frac{1}{2}+\frac{1}{2} \cos \left(2 \phi_{k}\right) e^{-2 \sigma_{\phi}^{2}}
$$

Eq. (19) follows by substituting (58) in (55), therefore concluding the proof.
APPENDIX D

ProOF OF LEMMA 3.5

The expectation of (8) is

$$
\begin{aligned}
\mathbb{E}\{ & \left.\hat{x}_{1, k+1 \mid k+1}\right\}=\left(1-\beta_{1, k}\right) x_{k+1}+\left(1-\beta_{1, k}\right) \mathbb{E}\left\{w_{x_{1, k+1}}^{(r)}\right\} \\
& +\beta_{1, k}\left(x_{k}+\mathbb{E}\left\{w_{x_{1, k}}\right\}+T V_{k} \cos \left(\phi_{k}\right) e^{-\frac{\sigma_{\phi}^{2}}{2}}\right) \\
= & \left(1-\beta_{1, k}\right) x_{k+1}+\beta_{1, k} x_{k+1}+\left(1-\beta_{1, k}\right) \mathbb{E}\left\{w_{x_{1, k+1}}^{(r)}\right\} \\
& +\beta_{1, k} \mathbb{E}\left\{w_{x_{1, k}}\right\}+\beta_{1, k} T V_{k} \cos \left(\phi_{k}\right)\left(e^{-\frac{\sigma_{\phi}^{2}}{2}}-1\right) \\
= & x_{k+1}+\mathbb{E}\left\{w_{x_{1, k+1}}\right\} .
\end{aligned}
$$

whereby the lemma follows.

\section{APPENDIX E}

\section{PROOF OF PROPOSITION 3.7}

The cost function in (11) is always positive for any choice of $\beta_{1, k} \in \mathbb{R}$ and of $\rho_{1, k}$, and it is a parabola in $\beta_{1, k}$, which is due to that $a_{k}>0$. It follows that the cost function is convex. Therefore, the optimal solution is given by computing the derivative, and observing that the optimal solution must lie in the interval $\mathscr{B}=[-1,1]$.

\section{APPENDIX F \\ PROOF OF LEMMA 4.1}

We note that the speed at time $k$ can be written as $V_{k}=V_{0}+$ $v_{3,1}+\ldots+v_{3, k-1}$. Since the process noise in the speed, $v_{3}$, is white, it follows that $V_{k} \sim \mathcal{N}\left(V_{0},(k-1) \sigma_{3}^{2}\right)$. Similarly, for the orientation $\phi_{k}$, we have that $\phi_{k} \sim \mathcal{N}\left(\phi_{0},(k-1) \sigma_{4}^{2}\right)$. Then the Lemma follows by using Lemma 3.4, after simple algebraic and trigonometric calculations.

\section{APPENDIX G}

\section{PROOF OF PROPOSITION 4.2}

First, we start by proving the following initial lemma:

Lemma G.1: Let $a, b$ be two independent random variables with non-zero average. Suppose that

$$
\lim _{k \rightarrow \infty} \frac{\mathbb{E}\left\{(b-\mathbb{E}\{b\})^{k}\right\}}{(\mathbb{E}\{b\})^{k}}=0 .
$$

Then,

$$
\mathbb{E}\left\{\frac{a}{b}\right\}=\frac{\mathbb{E}\{a\}}{\mathbb{E}\{b\}}\left[1+\sum_{k=1}^{\infty}(-1)^{k} \frac{\mathbb{E}\left\{(b-\mathbb{E}\{b\})^{k}\right\}}{(\mathbb{E}\{b\})^{k}}\right]
$$

Proof: Since $a$ and $b$ are independent, we have that

$$
\mathbb{E}\left\{\frac{a}{b}\right\}=\mathbb{E}\{a\} \mathbb{E}\left\{\frac{1}{b}\right\} .
$$

Furthermore,

$$
\mathbb{E}\left\{\frac{1}{b}\right\}=\mathbb{E}\left\{b^{-1}\right\}=\mathbb{E}\left\{\left(1+\frac{b-\mathbb{E}\{b\}}{\mathbb{E}\{b\}}\right)^{-1}\right\} \frac{1}{\mathbb{E}\{b\}} .
$$


By using Taylor series expansion as in [48, Section 5-4], we can write

$$
\mathbb{E}\left\{\left(1+\frac{b-\mathbb{E}\{b\}}{\mathbb{E}\{b\}}\right)^{-1}\right\}=1+\sum_{k=1}^{\infty}(-1)^{k} \frac{\mathbb{E}\left\{(b-\mathbb{E}\{b\})^{k}\right\}}{(\mathbb{E}\{b\})^{k}}
$$

The sum in the previous equation converges as a consequence of assumption (59). Finally, by substituting (62) in (61) and (60), the lemma follows.

Now, using Lemma G.1 above, we write the expectation as

$$
\begin{aligned}
& \mathbb{E}\left\{\frac{q^{2}}{q^{2}+z^{2}}\right\}=\mathbb{E}\left\{\frac{1}{1+\frac{z^{2}}{q^{2}}}\right\}=\frac{1}{1+\mathbb{E}\left\{\frac{z^{2}}{q^{2}}\right\}} \\
& \times\left[1+\sum_{k=1}^{\infty}(-1)^{k} \frac{\mathbb{E}\left\{\left(\frac{z^{2}}{q^{2}}-\mathbb{E}\left\{\frac{z^{2}}{q^{2}}\right\}\right)^{k}\right\}}{\left(1+\mathbb{E}\left\{\frac{z^{2}}{q^{2}}\right\}\right)^{k}}\right]
\end{aligned}
$$

We are left with the derivation of $\mathbb{E}\left\{z^{2} / q^{2}\right\}$. We start noting that, from Lemma G.1, we have

$$
\begin{aligned}
& \mathbb{E}\left\{\frac{z^{2}}{q^{2}}\right\}=\mathbb{E}\left\{z^{2}\right\} \mathbb{E}\left\{\frac{1}{q^{2}}\right\}=\frac{\mathbb{E}\left\{z^{2}\right\}}{\mathbb{E}\left\{q^{2}\right\}} \\
& \times\left[1+\sum_{k=1}^{\infty}(-1)^{k} \frac{\mathbb{E}\left\{\left(q^{2}-\mathbb{E}\left\{q^{2}\right\}\right)^{k}\right\}}{\left(\mathbb{E}\left\{q^{2}\right\}\right)^{k}}\right] .
\end{aligned}
$$

The variable $q^{2}$ has a non-central chi-squared distribution of 1 degree of freedom, thus $\mathbb{E}\left\{q^{2}\right\}=\sigma_{q}^{2}+\mu_{q}^{2}$, and

$$
\begin{aligned}
\mathbb{E}\left\{\left(q^{2}-\mathbb{E}\left\{q^{2}\right\}\right)^{k}\right\} & =\sigma_{q}^{2 k} \mathbb{E}\left\{\left(\frac{q^{2}}{\sigma_{q}^{2}}-\mathbb{E}\left\{\frac{q^{2}}{\sigma_{q}^{2}}\right\}\right)^{k}\right\} \\
& =\sigma_{q}^{2 k} \mu_{k} .
\end{aligned}
$$

This implies that

$$
\begin{gathered}
\lim _{k \rightarrow \infty} \frac{\mathbb{E}\left\{\left(q^{2}-\mathbb{E}\left\{q^{2}\right\}\right)^{k}\right\}}{\left(\mathbb{E}\left\{q^{2}\right\}\right)^{k}}=\lim _{k \rightarrow \infty} \frac{\sigma_{q}^{2 k} \mu_{k}}{\left(\mu_{q}^{2}+\sigma_{q}^{2}\right)^{k}} \\
=\lim _{k \rightarrow \infty} \frac{\mu_{k}}{\left(\frac{\mu_{q}^{2}}{\sigma_{q}^{2}}+1\right)^{k}}=0,
\end{gathered}
$$

since $\mu_{k}$ is bounded and the denominator grows unbounded, and condition (59) holds, which is needed for the convergence of the sum in Eq. (64). Therefore, Eq. (64) becomes

$$
\mathbb{E}\left\{\frac{z^{2}}{q^{2}}\right\}=\frac{\mu_{z}^{2}+\sigma_{z}^{2}}{\mu_{q}^{2}+\sigma_{q}^{2}}\left[1+\sum_{k=1}^{\infty}(-1)^{k} \frac{\sigma_{q}^{2 k} \mu_{k}}{\left(\mu_{q}^{2}+\sigma_{q}^{2}\right)^{k}}\right] .
$$

We are left with the computation of the expectation in the numerator of the summation in (63). This reduces to the k-th central moment of a doubly noncentral F-distribution. With this goal in mind, we first define $\chi_{z} \triangleq z^{2} / \sigma_{z}^{2}$ and $\chi_{q} \triangleq$ $q^{2} / \sigma_{q}^{2}$. We note that $\chi_{z}$ and $\chi_{q}$ are distributed according to a noncentral chi-squared distribution with 1 degree of freedom. Therefore $z^{2} / q^{2}=\chi_{z} / \chi_{q}$, since $\sigma_{z}^{2}=\sigma_{q}^{2}$, where the random variable $\chi_{z} / \chi_{q}$ is distributed according to a doubly noncentral F-distribution. Then, we have that

$$
\mathbb{E}\left\{\left(\frac{\chi_{z}}{\chi_{q}}-\mathbb{E}\left\{\frac{\chi_{z}}{\chi_{q}}\right\}\right)^{k}\right\}=\mu_{F, k} .
$$

The proposition follows by substituting (66) and (67) in (63), and noting that as required by (59),

$$
\lim _{k \rightarrow \infty} \frac{\mathbb{E}\left\{\left(\frac{z^{2}}{q^{2}}-\mathbb{E}\left\{\frac{z^{2}}{q^{2}}\right\}\right)^{k}\right\}}{\left(1+\mathbb{E}\left\{\frac{z^{2}}{q^{2}}\right\}\right)^{k}}=0,
$$

since the numerator is given by (67) and is bounded, whereas the denominator diverges because is the power of a number greater than 1 due to that the expectation in the denominator is positive.

\section{APPENDIX H}

\section{PROOF OF PROPOSITION 4.3}

The upper bound follows immediately from the fact that $q^{2} /\left(q^{2}+z^{2}\right) \leq 1, \forall q \in \mathbb{R}, \forall z \in \mathbb{R}$. Regarding the lower bound, by noting that $\ln (\cdot)$ is a concave function and applying Jensen's inequality, we obtain that

$$
e^{\mathbb{E}\left\{\ln \left(q^{2}\right)\right\}-\ln \left(\mathbb{E}\left\{q^{2}\right\}+\mathbb{E}\left\{z^{2}\right\}\right)} \leq \mathbb{E}\left\{\frac{q^{2}}{q^{2}+z^{2}}\right\} .
$$

Moreover, $\mathbb{E}\left\{\ln \left(q^{2}\right)\right\}=-\gamma_{e}-\ln (2)-2 \ln \left(\sigma_{q}\right)-$ $M\left(0, \frac{1}{2},-\mu_{q}^{2} / 2 \sigma_{q}^{2}\right)$, whereas the proposition follows by substituting the previous equation in (68).

\section{APPENDIX I}

\section{PROOF OF PROPOSITION 4.5}

We begin by observing that $\forall i, j$ the two matrices $\mathbf{J}^{(u b)}$ and $\mathbf{J}^{(l b)}$ satisfy $\mathbf{J}_{i j}^{(l b)} \leq \mathbf{J}_{i j} \leq \mathbf{J}_{i j}^{(u b)}$. Consider the element-wise upper bound $\mathbf{J}_{i j}^{(u b)}$. It follows that there exists a matrix $\mathbf{G}$ such that $\mathbf{J}_{i j}+\mathbf{G}_{i j} \geq \mathbf{J}_{i j}^{(u b)} \forall i, j$ and $\mathbf{J}+\mathbf{G}=\mathbf{J}^{(u b, G)}$, where $\mathbf{G}$ is symmetric and diagonally dominant with real and nonnegative elements. From the Gershgorin circle theorem, [49], it follows that all its eigenvalues are non-negative, i.e., $\lambda(\mathbf{G}) \geq$ 0 . Thus, $\lambda\left(\mathbf{J}^{(u b, G)}\right)=\lambda(\mathbf{J})+\lambda(\mathbf{G})$ because both $\mathbf{J}$ and $\mathbf{G}$ are Hermitian. Therefore, since $\mathbf{J}$ is positive semidefinite and $\lambda\left(\mathbf{J}^{(u b, G)}\right)>\lambda(\mathbf{J}), \mathbf{J} \preceq \mathbf{J}^{(u b, G)}$ and $\mathbf{0} \preceq \mathbf{J}^{(u b, G)}$. The proof is concluded by noting that the same steps may be employed for the lower bound $\mathbf{J}^{(l b, G)}$.

\section{REFERENCES}

[1] N. Patwari, J. N. Ash, S. Kyperountas, A. O. Hero III, R. L. Moses, and N. S. Correal, "Locating the nodes: cooperative localization in wireless sensor networks," IEEE Signal Processing Magazine, vol. 22, no. 4, pp. 54-69, July 2005.

[2] J. Rantakokko, J. Rydell, P. Strömbäck, P. Händel, J. Callmer, D. Törnqvist, F. Gustafsson, M. Jobs, and M. Gruden, "Accurate and reliable soldier and first responder indoor positioning: multisensor systems and cooperative localization," IEEE Wireless Communications, vol. 18, no. 2, pp. 10-18, Apr. 2011.

[3] H. Liu, H. Darabi, P. Banerjee, and J. Liu, "Survey of wireless indoor positioning techniques and systems," IEEE Transactions on Systems, Man, and Cybernetics, Part C: Applications and Reviews, vol. 37, no. 6 , pp. 1067-1080, Nov. 2007. 
[4] N. Patwari, I. Hero, A.O., M. Perkins, N. Correal, and R. O'Dea, "Relative location estimation in wireless sensor networks," IEEE Transactions on Signal Processing, vol. 51, no. 8, pp. 2137-2148, Aug. 2003.

[5] U. A. Khan, S. Kar, and J. M. F. Moura, "DILAND: An algorithm for distributed sensor localization with noisy distance measurements," IEEE Transactions on Signal Processing, vol. 58, no. 3, pp. 1940-1947, Mar. 2010.

[6] X. Chen, A. Edelstein, Y. Li, M. Coates, M. Rabbat, and A. Men, "Sequential Monte Carlo for simultaneous passive device-free tracking and sensor localization using received signal strength measurements," in 10th International Conference on Information Processing in Sensor Networks (IPSN), Apr. 2011, pp. 342-353.

[7] H. Wymeersch, J. Lien, and M. Win, "Cooperative localization in wireless networks," Proceedings of the IEEE, vol. 97, no. 2, pp. 427450, Feb. 2009.

[8] A. Conti, M. Guerra, D. Dardari, N. Decarli, and M. Win, "Network experimentation for cooperative localization," IEEE Journal on Selected Areas in Communications, vol. 30, no. 2, pp. 467-475, February 2012

[9] A. Bertrand and M. Moonen, "Seeing the bigger picture: How nodes can learn their place within a complex ad hoc network topology," IEEE Signal Processing Magazine, vol. 30, no. 3, pp. 71-82, 2013.

[10] M. Rabbat, R. Nowak, and J. Bucklew, "Robust decentralized source localization via averaging," in IEEE International Conference on Acoustics, Speech, and Signal Processing, vol. 5, 2005, pp. 1057-1060.

[11] U. Khan, S. Kar, and J. Moura, "Distributed sensor localization in random environments using minimal number of anchor nodes," IEEE Transactions on Signal Processing, vol. 57, no. 5, pp. 2000-2016, 2009.

[12] U. Hammes, E. Wolsztynski, and A. Zoubir, "Robust tracking and geolocation for wireless networks in NLOS environments," IEEE Journal of Selected Topics in Signal Processing, vol. 3, no. 5, pp. 889-901, 2009.

[13] J. Wang, Q. Gao, Y. Yu, H. Wang, and M. Jin, "Toward robus indoor localization based on bayesian filter using chirp-spread-spectrum ranging," IEEE Transactions on Industrial Electronics, vol. 59, no. 3, pp. 1622-1629, 2012.

[14] D. Zachariah, A. De Angelis, S. Dwivedi, and P. Handel, "Schedulebased sequential localization in asynchronous wireless networks," EURASIP Journal on Advances in Signal Processing, no. 1, p. 16, 2014 [Online]. Available: http://asp.eurasipjournals.com/content/2014/1/16

[15] S. Gezici, Z. Tian, G. Giannakis, H. Kobayashi, A. Molisch, H. Poor, and Z. Sahinoglu, "Localization via ultra-wideband radios: a look at positioning aspects for future sensor networks," IEEE Signal Processing Magazine, vol. 22, no. 4, pp. 70-84, July 2005.

[16] Y. Shen and M. Win, "Fundamental limits of wideband localization part I: A general framework," IEEE Transactions on Information Theory, vol. 56, no. 10 , pp. 4956-4980, Oct. 2010.

[17] M. Spirito, "On the accuracy of cellular mobile station location estimation," IEEE Transactions on Vehicular Technology, vol. 50, no. 3, pp. 674-685, May 2001

[18] B. Ristic and M. S. Arulampalam, "Tracking a manoeuvring target using angle-only measurements: algorithms and performance," Signal Processing, vol. 83, no. 6, pp. 1223-1238, June 2003.

[19] N. Bergman, "Recursive Bayesian estimation navigation and tracking applications," Ph.D. dissertation, Linköping Studies in Science and Technology, Sweden, 1999, dissertation n.579.

[20] L. Arienzo and M. Longo, "Posterior Cramér-Rao bound for rangebased target tracking in sensor networks," in IEEE/SP 15th Workshop on Statistical Signal Processing (SSP), Sept. 2009, pp. 541-544.

[21] K. Cheung, H. So, W.-K. Ma, and Y. Chan, "Received signal strength based mobile positioning via constrained weighted least squares," in IEEE International Conference on Acoustics, Speech, and Signal Processing (ICASSP), vol. 5, Apr. 2003, pp. V - 137-140.

[22] A. Speranzon, C. Fischione, K. Johansson, and A. SangiovanniVincentelli, "A distributed minimum variance estimator for sensor networks," IEEE Journal on Selected Areas in Communications, vol. 26, no. 4, pp. 609-621, May 2008.

[23] F. Gustafsson, Statistical sensor fusion. Studentliteratur, 2010.

[24] I. Skog and P. Händel, "In-car positioning and navigation technologies - a survey," IEEE Transactions on Intelligent Transportation Systems, vol. 10, no. 1, pp. 4-21, Mar. 2009.

[25] T. Kailath, A. H. Sayed, and B. Hassibi, Linear Estimation, 1st ed. Prentice Hall, Apr. 2000

[26] D. B. Jourdan, J. Deyst, J. J., M. Z. Win, and N. Roy, "Monte Carlo localization in dense multipath environments using UWB ranging," in IEEE International Conference on Ultra-Wideband (ICUWB), 5-8 Sept. 2005, pp. 314-319.
[27] J. Hol, F. Dijkstra, H. Luinge, and T. Schön, "Tightly coupled UWB/IMU pose estimation," in IEEE International Conference on Ultra-Wideband (ICUWB), Sept. 2009, pp. $688-692$

[28] A. De Angelis, J. O. Nilsson, I. Skog, P. Händel, and P. Carbone, "Indoor positioning by ultrawide band radio aided inertial navigation," Metrology and Measurement Systems, vol. XVII, pp. 447-460, 2010.

[29] R. Niu, R. Blum, P. Varshney, and A. Drozd, "Target localization and tracking in noncoherent multiple-input multiple-output radar systems," IEEE Transactions on Aerospace and Electronic Systems, vol. 48, no. 2 pp. $1466-1489$, april 2012.

[30] A. De Angelis, C. Fischione, and P. Händel, "A sensor fusion algorithm for mobile node localization," in 18th World Congress of the International Federation of Automatic Control (IFAC), Milan, Italy, Aug. 28 Sept. 22011.

[31] A. De Angelis and C. Fischione, "A distributed information fusion method for localization based on Pareto optimization," in 7th IEEE International Conference on Distributed Computing in Sensor Systems (DCOSS), Barcelona, Spain, June 27-29 2011.

[32] P. Tichavský, C. Muravchik, and A. Nehorai, "Posterior Cramér-Rao bounds for discrete-time nonlinear filtering," IEEE Transactions on Signal Processing, vol. 46, no. 5, pp. 1386-1396, May 1998

[33] A. De Angelis, M. Dionigi, R. Moschitta, A. Giglietti, and P. Carbone, "Characterization and modeling of an experimental UWB pulse-based distance measurement system," IEEE Transactions on Instrumentation and Measurement, vol. 58, no. 5, pp. 1479-1486, May 2009.

[34] M. Gholami, S. Gezici, and E. Strom, "Tdoa based positioning in the presence of unknown clock skew," Communications, IEEE Transactions on, vol. 61, no. 6, pp. 2522-2534, June 2013.

[35] A. Mourikis and S. I. Roumeliotis, "Performance analysis of multirobot cooperative localization," IEEE Transactions on Robotics, vol. 22, no. 4, pp. 666-681, Aug. 2006

[36] A. Georgiev and P. K. Allen, "Localization methods for a mobile robot in urban environments," IEEE Transactions on Robotics, vol. 20, no. 5 , pp. 851-864, Oct. 2004.

[37] S. Boyd and L. Vandenberghe, Convex Optimization. Cambridge University Press, 2004

[38] I. Guvenc, S. Gezici, and Z. Sahinoglu, "Fundamental limits and improved algorithms for linear least-squares wireless position estimation," Wireless Communications and Mobile Computing, 2010.

[39] S. M. Kay, Fundamentals of Statistical Signal Processing: Estimation Theory. Englewood Cliffs, NJ: Prentice Hall, 1993.

[40] J. Taylor, "The Cramér-Rao estimation error lower bound computation for deterministic nonlinear systems," IEEE Transactions on Automatic Control, vol. 24, no. 2, pp. 343-344, Apr. 1979

[41] B. Ristic, S. Arulampalam, and N. Gordon, Beyond the Kalman Filter: Particle Filters for Tracking Applications. Artech House, 2004.

[42] T. Bréhard and J.-P. Le Cadre, "Closed-form posterior Cramér-Rao bound for bearings-only tracking," IEEE Transactions on Aerospace and Electronic Systems, vol. 42, no. 4, pp. 1198-1223, 2006.

[43] A. Bishop, B. Fidan, B. Anderson, K. Dogancay, and P. Pathirana, "Optimality analysis of sensor-target localization geometries," Automatica, vol. 46, no. 3, pp. 479-492, Mar. 2010.

[44] I. Shames, B. Fidan, B. Anderson, and H. Hmam, "Cooperative selflocalization of mobile agents," IEEE Transactions on Aerospace and Electronic Systems, vol. 47, no. 3, pp. 1926-1947, July 2011.

[45] M. Abramowitz and I. A. Stegun, Handbook of Mathematical Functions with Formulas, Graphs, and Mathematical Tables. Dover Publications, 1972.

[46] E. Abbott and D. Powell, "Land-vehicle navigation using GPS," Proceedings of the IEEE, vol. 87, no. 1, pp. 145-162, Jan. 1999.

[47] S. Julier and J. Uhlmann, "Unscented filtering and nonlinear estimation," Proceedings of the IEEE, vol. 92, no. 3, pp. 401-422, Mar. 2004.

[48] A. Papoulis, Probability, Random Variables and Stochastic Processes. McGraw-Hill Companies, February 1991.

[49] R. Horn and C. Johnson, Matrix analysis. Cambridge University Press, 1990 . 